\title{
Distinct subpopulations of FOXD1 stroma-derived cells regulate renal erythropoietin
}

\author{
Hanako Kobayashi, ${ }^{1,2}$ Qingdu Liu, ${ }^{1,2}$ Thomas C. Binns, ${ }^{2}$ Andres A. Urrutia, ${ }^{2}$ Olena Davidoff,, ${ }^{1,2}$ Pinelopi P. Kapitsinou, ${ }^{2}$ \\ Andrew S. Pfaff, ${ }^{2}$ Hannes Olauson, ${ }^{3}$ Annika Wernerson, ${ }^{3,4}$ Agnes B. Fogo, ${ }^{5}$ Guo-Hua Fong, ${ }^{6}$ \\ Kenneth W. Gross, ${ }^{7}$ and Volker H. Haase ${ }^{1,2,8}$
}

${ }^{1}$ Medical and Research Services, Department of Veterans Affairs Hospital, Tennessee Valley Healthcare System, Nashville, Tennessee, USA. ${ }^{2}$ Department of Medicine, Vanderbilt University School of Medicine,

Nashville, Tennessee, USA. ${ }^{3}$ Division of Renal Medicine, Department of Clinical Sciences, Intervention and Technology, and ${ }^{4}$ Clinical Pathology and Cytology, Karolinska University Hospital,

Karolinska Institutet, Stockholm, Sweden. 5Department of Pathology, Microbiology and Immunology and Department of Pediatrics, Vanderbilt University School of Medicine, Nashville, Tennessee, USA.

${ }^{6}$ Center for Vascular Biology, Department of Cell Biology, University of Connecticut Health Center, Farmington, Connecticut, USA. Department of Molecular and Cellular Biology, Roswell Park Cancer Institute,

Buffalo, New York, USA. ${ }^{8}$ Departments of Cancer Biology, and Molecular Physiology and Biophysics, Vanderbilt University School of Medicine, Nashville, Tennessee, USA.

\begin{abstract}
Renal peritubular interstitial fibroblast-like cells are critical for adult erythropoiesis, as they are the main source of erythropoietin (EPO). Hypoxia-inducible factor 2 (HIF-2) controls EPO synthesis in the kidney and liver and is regulated by prolyl-4-hydroxylase domain (PHD) dioxygenases PHD1, PHD2, and PHD3, which function as cellular oxygen sensors. Renal interstitial cells with EPO-producing capacity are poorly characterized, and the role of the PHD/HIF-2 axis in renal EPO-producing cell (REPC) plasticity is unclear. Here we targeted the PHD/HIF-2/EPO axis in FOXD1 stroma-derived renal interstitial cells and examined the role of individual PHDs in REPC pool size regulation and renal EPO output. Renal interstitial cells with EPO-producing capacity were entirely derived from FOXD1-expressing stroma, and Phd2 inactivation alone induced renal Epo in a limited number of renal interstitial cells. EPO induction was submaximal, as hypoxia or pharmacologic PHD inhibition further increased the REPC fraction among Phd2 ${ }^{-/-}$renal interstitial cells. Moreover, Phd1 and Phd3 were differentially expressed in renal interstitium, and heterozygous deficiency for Phd1 and Phd 3 increased REPC numbers in Phd2-/- mice. We propose that FOXD1 lineage renal interstitial cells consist of distinct subpopulations that differ in their responsiveness to Phd2 inactivation and thus regulation of HIF-2 activity and EPO production under hypoxia or conditions of pharmacologic or genetic PHD inactivation.
\end{abstract}

\section{Introduction}

The hypoxic induction of erythropoietin (EPO), a hypoxia-inducible factor-regulated (HIF-regulated) glycoprotein hormone that is essential for normal erythropoiesis, represents one of the most sensitive systemic hypoxia responses in humans (1). In the bone marrow, EPO acts on CFU, pro-, and early basophilic erythroblasts and inhibits apoptosis of red cell precursors, which increases rbc mass and thus oxygen-carrying capacity in blood. In adults, the major site of EPO synthesis is the kidney, where peritubular interstitial fibroblast-like cells respond to decreases in tissue $\mathrm{pO}_{2}$ with increased EPO synthesis. Abnormal EPO responsiveness in the bone marrow or deregulated renal EPO production can lead either to excessive rbc production and polycythemia, or to hypoproliferative anemia, a condition that is commonly found in patients with chronic kidney disease (CKD) and is primarily due to relative EPO deficiency (1).

HIF-2, the transcription factor responsible for the hypoxic induction of renal EPO, is required for normal erythropoiesis (2), as its deletion from renal tissue results in severe anemia (3). HIFs consist of an oxygen-sensitive $\alpha$-subunit and a constitutively

Conflict of interest: V.H. Haase serves on the Scientific Advisory Board of Akebia Therapeutics, a company that develops prolyl-4-hydroxylase inhibitors for the treatment of anemia.

Submitted: July 10, 2015; Accepted: March 1, 2016

Reference information: / Clin Invest. 2016;126(5):1926-1938. doi:10.1172/JCI83551. expressed $\beta$-subunit, HIF- $\beta$, which is also known as the aryl hydrocarbon receptor nuclear translocator (ARNT). Together with HIF-1, HIF-2 regulates a multitude of hypoxia responses that allow cells to adapt to and survive low-oxygen environments (4). While HIF- $\alpha$ subunits are continuously synthesized, they are rapidly degraded in the presence of molecular oxygen. Under normoxia, oxygen-, iron-, and 2-oxoglutarate-dependent prolyl-4-hydroxylase domain (PHD) proteins, PHD1, PHD2, and PHD3, also known as egl-9 homolog 2 (EGLN2), EGLN1, and EGLN3, respectively, hydroxylate HIF- $\alpha$ at specific proline residues. This hydroxylation reaction is key to targeting HIF- $\alpha$ for proteasomal degradation via ubiquitylation by the von Hippel-Lindau (VHL) E3-ubiquitin ligase complex (5). Under hypoxia prolyl-4-hydroxylation of HIF- $\alpha$ is inhibited, resulting in its translocation to the nucleus, where it heterodimerizes with ARNT and transactivates a large number of oxygen-regulated genes (5). In the kidney, activation of HIF-2 by either hypoxia or pharmacologic or genetic PHD inhibition increases serum EPO levels and rbc production (1). However, the role of individual PHDs in the regulation of HIF-2-mediated renal hypoxia responses and EPO gene transcription under physiologic and injury conditions is not well understood.

IHC and in situ hybridization (ISH) studies as well as findings from genetic mouse models have provided strong evidence that fibroblast-like interstitial cells and not epithelial or endothelial cells synthesize EPO in the kidney (6-9). Renal interstitial fibro- 
A

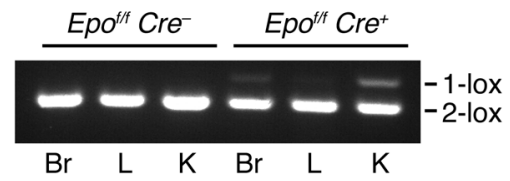

B
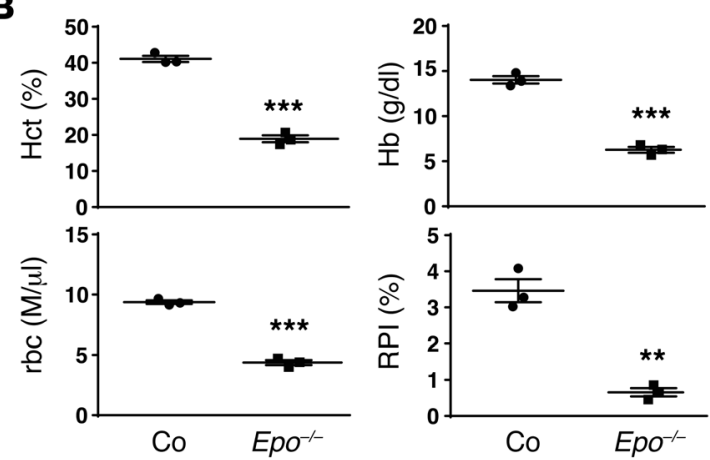

C
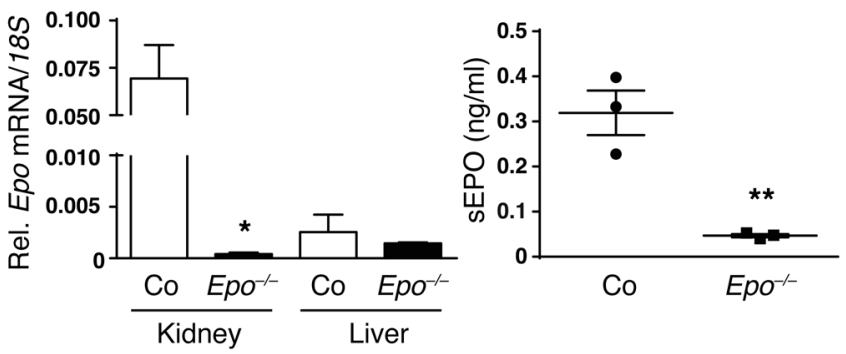

blast-like cells encompass a heterogeneous cell population that consists of perivascular fibroblast-like cells and pericytes $(10,11)$. Under hypoxic conditions the number of renal EPO-producing cells (REPCs; this term refers to cells that actively synthesize EPO) increases in a tissue $\mathrm{pO}_{2}$ - and HIF-2-dependent manner and determines renal EPO output and thus plasma EPO levels $(8,12)$. This increase in REPC numbers is furthermore associated with morphologic changes such as enlargement of peritubular space, which includes increases in both interstitial and capillary volume (13).

Despite advances in understanding HIF-dependent regulation of renal EPO production, the physiologic behavior of REPCs remains poorly characterized, and the degree of cellular and molecular heterogeneity within this cell population has not been defined. Although genetic cell fate analysis using myelin protein zero-Cre $(\mathrm{PO}-\mathrm{Cre})$ has indicated that a subpopulation of REPCs may originate from migrating neural crest cells (14), a common progenitor cell from which REPCs are derived has not been identified. REPCs have a unique morphologic appearance as they are characterized by dendrite-like cellular processes and the expression of PDGF receptor- $\beta$ (PDGFRB) and ecto-5'-nucleotidase (CD73), cell surface markers that are also found in pericytes, suggesting that renal pericytes have the capacity to synthesize EPO when stimulated by hypoxia (1). However, the degree to which renal interstitial fibroblasts and pericytes contribute to the renal EPO response is not known.

During kidney development, FOXD1-expressing stromal cells surround the cap mesenchyme and give rise to cortical and medullary renal interstitial fibroblasts, pericytes, mesangial cells, and vascular smooth muscle cells (VSMCs) (11). In order to examine the role of individual PHDs in the regulation of renal interstitial
Figure 1. Inactivation of Epo in FOXD1 stroma-derived cells results in severe anemia. (A) PCR analysis of genomic brain (Br), liver ( $L$ ), and kidney (K) DNA isolated from control $\left(E p o^{f / f f l} \mathrm{Cre}^{-}\right)$and Foxd1-Epo-/- $\left(\mathrm{Epo}^{\mathrm{fl/fl}} \mathrm{Cre}^{+}\right)$ mutant mice. 2-lox denotes the nonrecombined conditional allele and 1-lox the recombined allele. (B) Values for hematocrit ( $\mathrm{Hct}$ ), hemoglobin $(\mathrm{Hb})$, rbc counts, and reticulocyte production index (RPI) from individual control (Co) and Foxd1- $\mathrm{Epo}^{-/-}\left(\mathrm{Epo}^{-/-}\right)$mutant mice ( $n=3$ each). (C) Renal and hepatic Epo mRNA and serum EPO (sEPO) levels from control and Foxd1-Epo-/- mutant mice $(n=3)$. Data are represented as mean \pm SEM; 2-tailed Student's $t$ test; ${ }^{*} P<0.05,{ }^{* *} P<0.01,{ }^{* * *} P<0.001$ compared with control group.

HIF-2 activity, and to determine to what degree pericytes and other perivascular cells contribute to the kidney's EPO response, we have used the Cre-loxP system to target the PHD/HIF-2/EPO axis in FOXD1 stroma-derived renal interstitium. We found that the ability to synthesize EPO is completely contained within the FOXD1 stroma-derived cell population and that the majority of FOXD1-derived interstitial cells in cortex and outer medulla have EPO-producing capacity. Using multiplex ISH techniques we quantified the number of EPO-producing cells and determined the size of the REPC pool under conditions of systemic hypoxia or individual HIF-PHD inactivation. Our data provide genetic evidence for the existence of distinct subpopulations of renal interstitial cells that differ in responsiveness to $P h d 2$ inactivation and thus regulation of HIF-2 activity and EPO synthesis.

\section{Results}

REPCs are derived from FOXD1-expressing stroma. We have previously examined the role of HIF-2 $\alpha$ in renal EPO production using P3Pro-Cre transgenic mice, which express Cre-recombinase in both renal tubular epithelium and interstitium (3). To specifically investigate the role of PHD oxygen sensors in Epo regulation in renal interstitium, we used Foxd $1^{\mathrm{Cr} / \mathrm{t}}$ transgenic mice and targeted the components of the PHD/HIF-2/EPO axis individually or in combination. The Foxd1-Cre transgene encodes an EGFP/Cre-recombinase (EGFP/Cre) fusion protein under transcriptional control of the Foxd1 promoter and was generated by homologous recombination. Foxd1-Cre is expressed during kidney development, but not in the adult kidney $(15,16)$. To visualize FOXD1 stroma-derived interstitial cells in the kidney, Foxd $1^{\mathrm{Cre} /}+$ transgenic mice were intercrossed with Cre-reporter mice (ROSA26-ACTB-tdTomato,-EGFP), which expressed fluorescent membrane-bound tdTomato red prior to excision and membrane-bound EGFP following excision of a floxed stop cassette (17). As expected, EGFP expression was observed in the interstitial space of cortex and medulla, as well as in cells of vessel walls and glomeruli (Supplemental Figure 1A; supplemental material available online with this article; doi:10.1172/JCI83551DS1). IHC analysis revealed a normal pattern of PDGFRB expression, indicating that kidney development was not affected (Supplemental Figure 1B). EGFP expression was furthermore observed in a subpopulation of tubular epithelial cells, which is consistent with previously published reports (16). The latter is most likely due to FOXD1 expression in a small number of mesenchymal progenitor cells that give rise to both FOXD1-expressing stroma and SIX2-expressing epithelial progenitors cells (16).

In order to study the degree of contribution of FOXD1 stromaderived interstitial cells to EPO production in the kidney, we generated Foxd1 ${ }^{\mathrm{Cre} /+} E \mathrm{E} 0^{f / / l}$ mice, herein referred to as Foxd1-Epo ${ }^{-/}$, by 
A
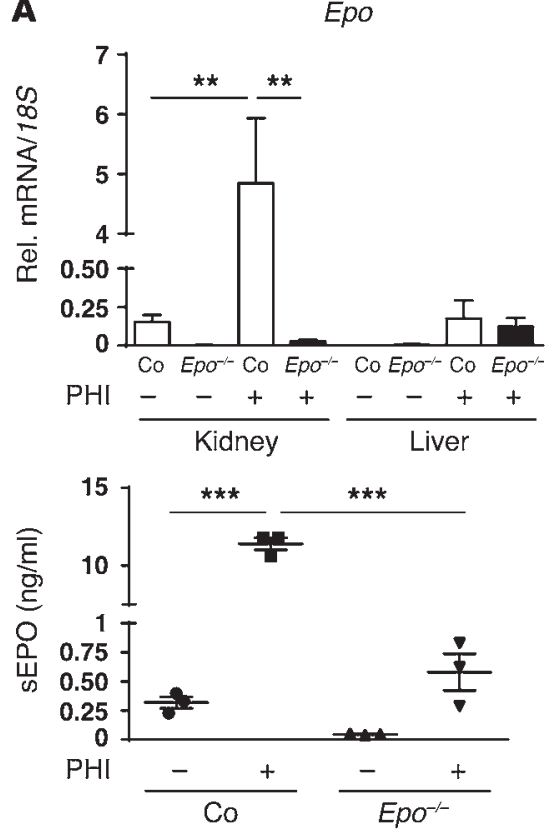

B
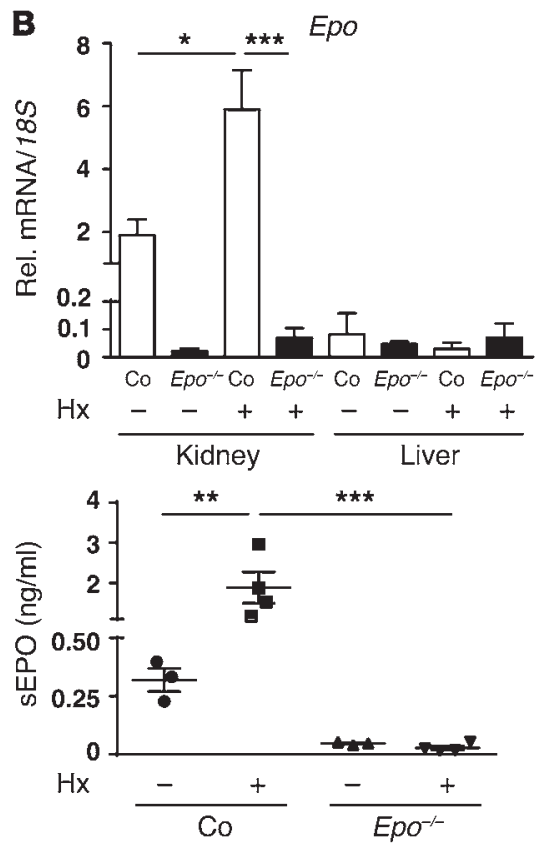

Figure 2. Renal EPO-producing cells are derived from FOXD1 stromal progenitors. Shown are the relative renal and liver Epo responses to treatment with an orally administered HIF-PHD inhibitor (PHI) or normobaric hypoxia in control (Co) and Foxd1-Epo-1mutants $\left(\mathrm{Epo}^{-/-}\right)$. (A) PHI treatment with compound GSK1002083A ( $n=3$ each). (B) Treatment with 2 days of normobaric hypoxia $(\mathrm{Hx})$ at $10 \% \mathrm{O}_{2}(n=3$ and 4 , respectively). The induction of Epo mRNA is completely suppressed (top panels), and serum EPO responses (bottom panels) are blunted in Foxd1-Epo-1- mutants. Data are represented as mean \pm SEM; 2-way ANOVA with post hoc Tukey's test; ${ }^{*} P<0.05,{ }^{*} P<0.01$, ${ }^{* *} P<0.001$. crossing Foxd $1^{\text {Cre/+ }}$ transgenics with mice homozygous for a conditional Epo allele (18). These mice were born in mendelian ratios and developed normally. Genomic PCR was used to assess recombination in different tissues. Recombination was detected in multiple tissues including kidney and brain, but not in the liver (Figure 1A). Foxd1-Epo ${ }^{-/-}$mice developed severe anemia (hematocrit [Hct] of $18.9 \% \pm 1.0 \%$ vs. $41.1 \% \pm 0.9 \%$ in controls; hemoglobin [Hb] of $6.3 \pm 0.3 \mathrm{~g} / \mathrm{dl}$ vs. $14.0 \pm 0.4 \mathrm{~g} / \mathrm{dl}$ and $\mathrm{rbc}$ count of $4.4 \pm 0.2 \mathrm{M} / \mu \mathrm{l}$ vs. $9.4 \pm 0.2 \mathrm{M} / \mu \mathrm{l}$ in controls; $n=3$ each; $P<0.001$ each; Figure 1B), which was associated with a decreased reticulocyte production index (0.7 \pm 0.1 in mutants vs. $3.5 \pm 0.3$ in controls; $n=3$ each; $P<$ 0.01), indicating that Foxd1-Epo ${ }^{-/-}$mice had developed hypoproliferative anemia (Figure 1B). We next used real-time PCR to determine renal Epo transcript levels, which were severely reduced. The absence of significant renal Epo transcription in mutant mice was also reflected in abnormal plasma EPO concentrations, as serum EPO levels were significantly lower in mutant mice $(0.047 \pm 0.004$ $\mathrm{ng} / \mathrm{ml}$ vs. $0.32 \pm 0.059 \mathrm{ng} / \mathrm{ml}$ in controls; $n=3 ; P<0.01 ;$ Figure $1 \mathrm{C})$.

Since our data suggested that the renal EPO response was completely ablated in anemic Foxd1-Epo-/ mice, we examined whether exposure to normobaric hypoxia $\left(10 \% \mathrm{O}_{2}\right.$ for 2 days) or treatment with an oral pan-PHD inhibitor (PHI), GSK1002083A, which has been shown to robustly activate HIF-2 in the kidney (3), was capable of stimulating EPO responses in Foxd1-Epo-/kidneys. While PHI or hypoxia treatment increased renal Epo mRNA levels in $C r e^{-}$control mice ( 32 -fold increase in the PHI group and $\sim 3$-fold for the hypoxia group compared with nontreated $\mathrm{Cre}^{-}$controls; Figure 2, A and B), significant increases in renal Epo mRNA levels were not detected in PHI- or hypoxia-treated Foxd1-Epo ${ }^{-/-}$mutants. Serum EPO levels in $\mathrm{Cre}^{-}$control mice increased from $0.32 \pm 0.059 \mathrm{ng} / \mathrm{ml}$ to $12.7 \pm 0.9 \mathrm{ng} / \mathrm{ml}$ with PHI treatment and to $1.9 \pm 0.4 \mathrm{ng} / \mathrm{ml}$ following exposure to hypoxia ( $n=3 ; P<0.001$ and 0.01, respectively). In Foxd1-Epo ${ }^{-/-}$mutant mice hypoxia treatment did not result in an increase in serum EPO $(n=4)$, while PHI treatment raised serum EPO concentra- tion from $0.047 \pm 0.004 \mathrm{ng} / \mathrm{ml}$ to $0.58 \pm 0.16 \mathrm{ng} / \mathrm{ml}(n=3)$. The latter is most likely a reflection of increased liver Epo transcription that is expected with PHI treatment or severe hypoxic anemia (3). To provide additional evidence that the EPO-producing ability of the kidney is completely contained within the FOXD1 lineage cells, we inactivated Hif2a using Foxd1-Cre, as HIF-2 is the major regulator of renal Epo transcription (3); conditional knockout mice are herein referred to as Foxd1-Hif $2 a^{-/-}$mutants. Similarly to Foxd1-Epo ${ }^{-/}$mice, Foxd1-Hif2 $a^{-/}$mutants developed severe anemia and did not respond to PHI treatment with increased renal Epo transcription (Supplemental Figure 2). Taken together, our genetic data provide strong evidence that cells that produce EPO are exclusively derived from FOXD1-expressing renal stroma and that the EPO-producing ability of the kidney in response to hypoxia challenge or pharmacologic HIF activation is completely contained within FOXD1 lineage cells.

Inactivation of PHD2, but not PHD1 or PHD3, induces EPO in FOXD1 lineage cells. Inactivation of all 3 PHDs is required for a strong and sustained EPO production in the liver (19-23). In order to investigate the role of individual PHDs in the regulation of renal EPO, we generated Foxd $1^{\mathrm{Cr} /+} \mathrm{Phd} 1^{\mathrm{fl} / f l}$, Foxd $1^{\mathrm{Cre} / \mathrm{+}} \mathrm{Ph} d 2^{\mathrm{flfl} \text {, }}$, and Foxd $1^{\text {Cre/ }}$ Phd $3^{\text {fl/fl }}$ mice, herein referred to as Foxd1-Phd1--, Foxd1Phd 2 ${ }^{--}$, and Foxd1-Phd3 ${ }^{-/-}$mutants, respectively. Genomic PCR was used to confirm efficient recombination in the kidney (shown here for Foxd1-Phd2---; Figure 3A). Inactivation of PHD1 and PHD3 alone or together (Foxd1 $1^{\text {Cre/+ }} P h d 1^{f / f l} P h d 3^{f / f l}$ ) did not change rbc parameters or induce renal Epo transcription (Supplemental Figure 3). In contrast, Foxd1-Phd2-/- mutants developed polycythemia. By 6 weeks of age Foxd1-Phd $2^{-/-}$mutants were characterized by a significant elevation in rbc numbers (Hct of $62.8 \% \pm 1.0 \%$ vs. $45.8 \% \pm 1.1 \%$ in controls; $\mathrm{Hb}$ of $18.5 \pm 0.4 \mathrm{~g} / \mathrm{dl}$ vs. $15.6 \pm 0.3 \mathrm{~g} / \mathrm{dl}$ in controls; rbc counts of $15.1 \pm 0.5 \mathrm{M} / \mu \mathrm{l}$ vs. $9.8 \pm 0.2 \mathrm{M} / \mu \mathrm{l}$ in controls; $n=6$ and 11, respectively; $P<0.001$; Figure $3 \mathrm{~B}$ ). This was associated with a significant increase in reticulocyte production index (Figure 3B), increased renal Epo mRNA expression ( 206-fold 
A

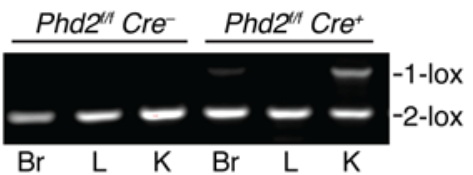

B
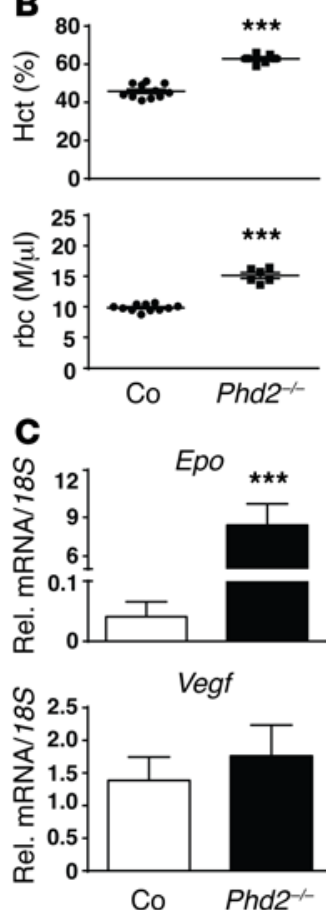
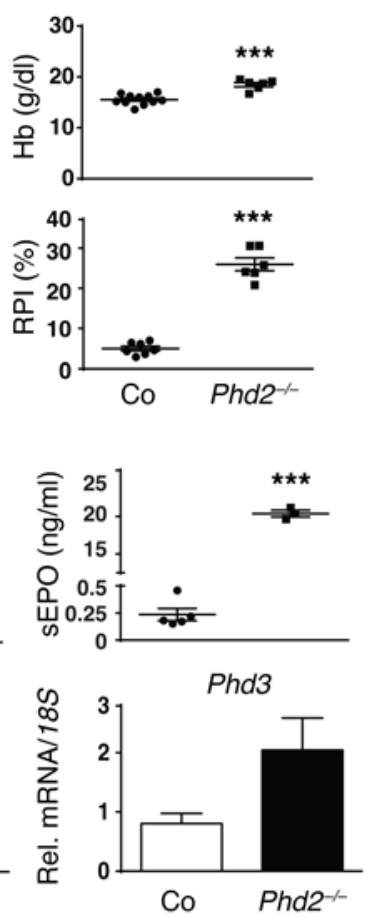

D

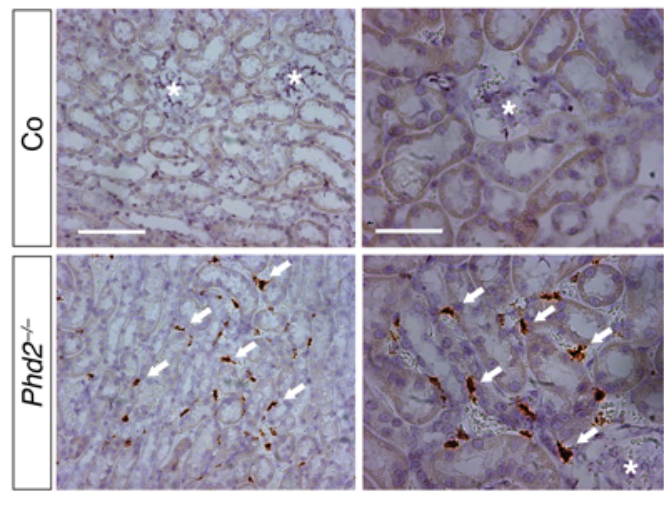

E

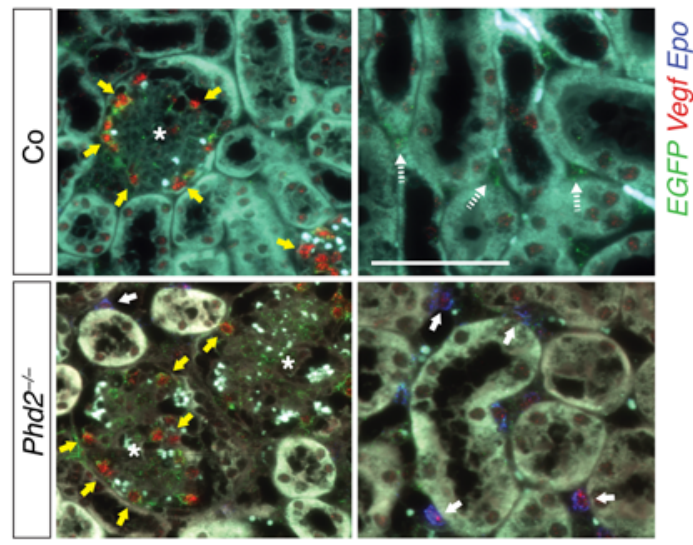

Figure 3. Inactivation of Phd2 in FOXD1 stroma-derived renal interstitial cells results in polycythemia. (A) PCR analysis of genomic brain (Br), liver (L),

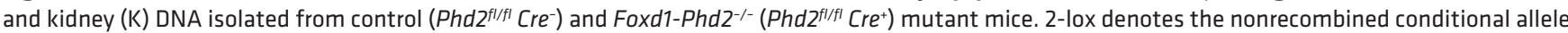
and 1-lox the recombined allele. (B) Individual values for hematocrit (Hct), hemoglobin ( $\mathrm{Hb}$ ), rbc counts, and reticulocyte production index (RPI) for control (Co) and Foxd1-Phd2 $2^{--}\left(P h d 2^{-/-}\right)$mutant mice $(n=6-11)$. Data are represented as mean \pm SEM; 2-tailed Student's $t$ test; ${ }^{* *} P<0.001$. (C) Renal Epo, Vegf, and Phd3 mRNA and serum EPO (sEPO) level from control and Foxd1-Phd2 $2^{--}$mutant mice $(n=3-6)$. Graph bars represent mean \pm SEM; 2 -tailed Student's $t$ test; ${ }^{* *} P<0.001$ compared with control group. (D) Epo ISH in formalin-fixed, paraffin-embedded kidney sections from control and Foxd1-Phd2-/- kidneys using an HRP-based colorimetric detection method. White arrows indicate positively stained cells and asterisks depict glomeruli. Scale bars: $100 \mu \mathrm{m}$

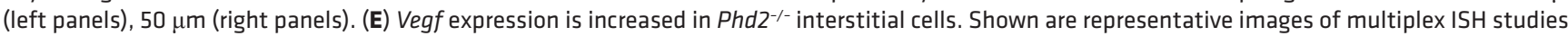
using formalin-fixed, paraffin-embedded kidney tissue sections from Foxd1-mT/mG (Co) and Foxd1-mT/mG-Phd2 ${ }^{-/-}\left(P^{2} d 2^{-/-}\right)$mice. Epo $^{+}$Vegf ${ }^{+}$cells are denoted by white arrows; yellow arrows depict Vegf-expressing glomerular cells; glomeruli are marked with asterisks. Scale bar: $50 \mu \mathrm{m}$ (all panels).

compared with $C r e^{-}$controls; $n=6$ each; $P<0.001 ;$ Figure 3 C), and elevated serum EPO levels $(20.4 \pm 0.4 \mathrm{ng} / \mathrm{ml}$ vs. $0.24 \pm 0.06 \mathrm{ng} /$ $\mathrm{ml}$ in controls; $n=3$ and 5 , respectively; $P<0.001$; Figure 3 C). By 8 weeks of age Hct was increased to $81.3 \% \pm 3.2 \%$ in Foxd1-Phd $2^{--}$ mutant mice compared with $48.0 \% \pm 0.5 \%$ in control mice $(n=$ 3 and 5, respectively; data not shown). An increase in other HIFregulated genes such as Vegf and Phd 3 was not observed in wholekidney homogenates from Foxd1-Phd2 ${ }^{--}$mutants (Figure 3C). Since PHD2 inactivation inhibits the degradation of both HIF-1 $\alpha$ and HIF- $2 \alpha$, we examined HIF- $1 \alpha$ and HIF- $2 \alpha$ protein expression by immunoblot and IHC. While we detected increased HIF-2 $\alpha$ protein levels in total tissue homogenates from Foxd1-Phd $2^{-/-}$kidneys by IB, HIF-1 $\alpha$ protein levels did not differ from those of control animals (Supplemental Figure 4). As expected, HIF-2 $\alpha$ localized to the renal interstitium as well as glomerular cells (Supplemental Figure 4), while Epo mRNA was only detectable in cortical and outer medullary peritubular interstitial cells by ISH using an HRPbased chromogenic detection method (Figure 3D). In contrast to whole-tissue homogenates, the expression of HIF target gene Vegf was increased in renal interstitium, where the majority of $\mathrm{Epo}^{+}$ cells expressed detectable levels of Vegf (Figure 3E). In summary, our data demonstrate that inactivation of PHD2, but not PHD1 or PHD3, is sufficient for the activation and induction of EPO synthesis in FOXD1 stroma-derived renal interstitial cells.

Epo induction in Foxd1-Phd2-- kidneys is submaximal. Although Foxd1-Cre-mediated inactivation of $P h d 2$ alone resulted in a very strong increase in renal Epo mRNA levels, it was unclear whether Epo was maximally induced. In order to address this question, we generated Foxd $1^{\text {Crel }}+$ ROSA26-ACTB-tdTomato,-EGFP Phd $2^{A / A l}$ conditional knockout mice, herein referred to as Foxd1- $m T / m G$-Phd2 - mutants. In this knockout line, Phd2-deficient cells were simultaneously tagged with membrane-bound EGFP, which permitted identification of cells having undergone recombination by visual inspection.

We first used high-resolution ISH to visualize EGFP transcripts in formalin-fixed, paraffin-embedded kidney sections. Consistent with the results from fluorescence microscopy and previous reports, EGFP-expressing cells were detected in renal interstitium, in vessel walls, in glomeruli, and in a small subpopulation 

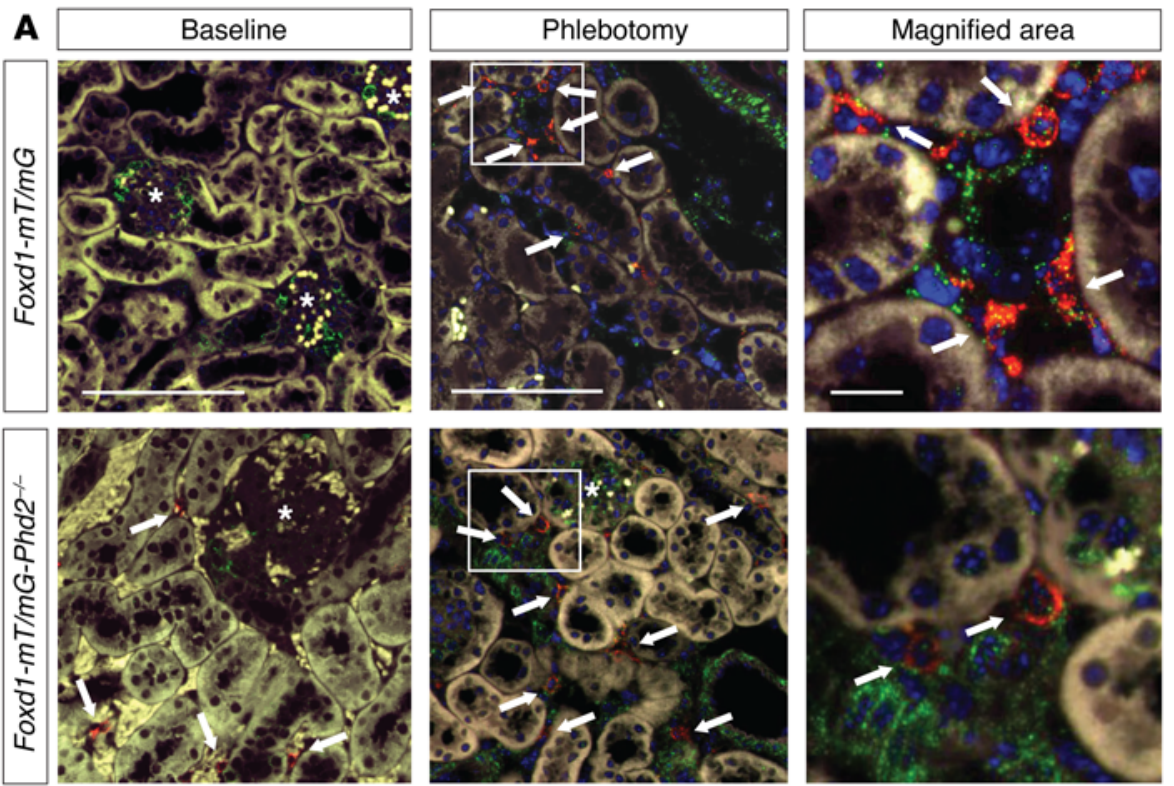

B
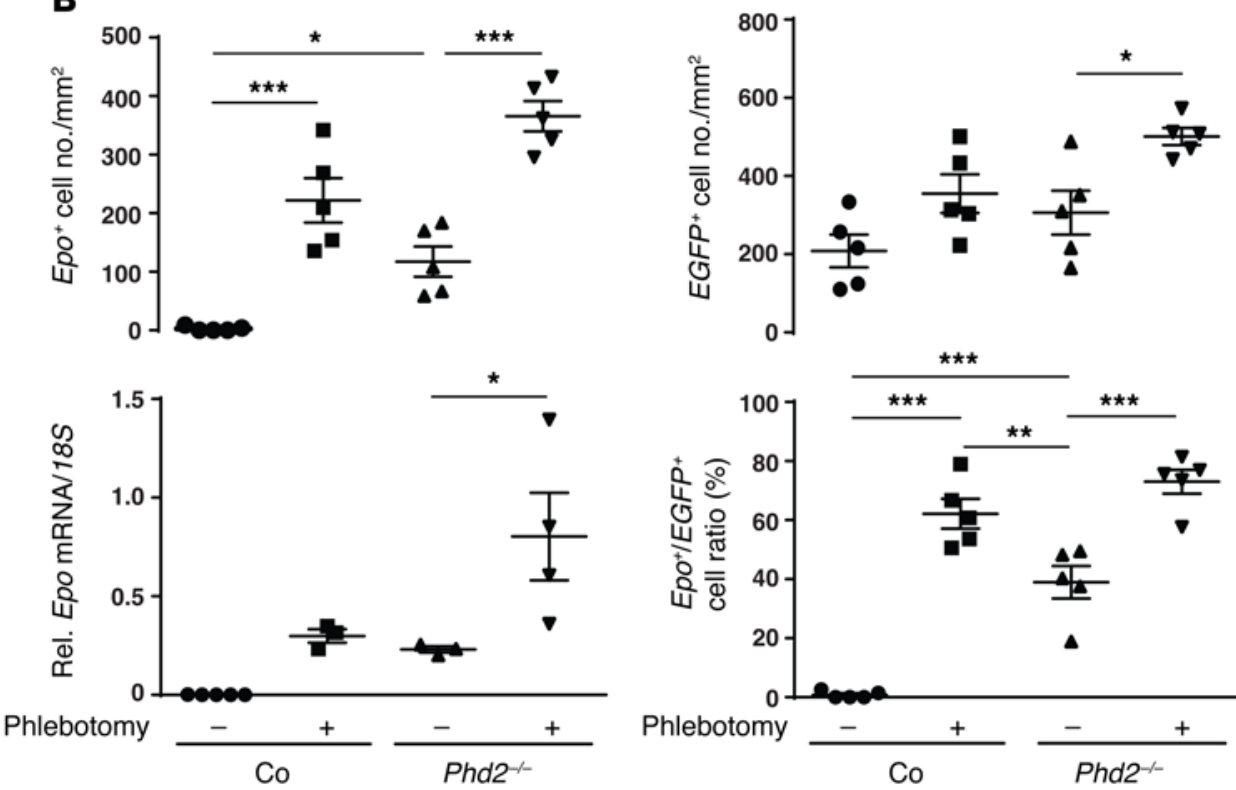

Figure 4. Epo induction in Foxd1-Phd2-1mutants is submaximal. Shown are the results of multiplex ISH for Epo and EGFP RNA using formalin-fixed, paraffinembedded kidney tissue sections from Foxd1-mT/mG (Co) and Foxd1-mT/mGPhd2 $2^{--}\left(\right.$Phd2 $\left.2^{--}\right)$mutant mice at baseline and after phlebotomy ( $n=5$ for each group). (A) Representative images of kidney tissue sections containing peritubular interstitial cells expressing Epo (red signal) and/or EGFP (green signal). Nuclei are stained with DAPI (blue signal). Scale bars: $100 \mu \mathrm{m}$ (baseline and phlebotomy), $10 \mu \mathrm{m}$ (high-magnification images). White arrows identify Epo-expressing cells; asterisks depict glomeruli. (B) Top left panel: Quantification of Epo-expressing cells. Shown is the absolute number of Epo+ cells per square millimeter of kidney tissue. Bottom left panel: Epo mRNA levels relative to 185 in total kidney tissue homogenates as determined by real-time PCR $(n=3-5)$. Top right panel: Quantification of EGFP-expressing cells. Shown is the absolute number of EGFP' cells per square millimeter. Bottom right panel: Fraction of Epo-expressing cells among EGFP+ cells. Data are represented as mean $\pm \mathrm{SEM}$; 2-way ANOVA with post hoc Tukey's test; ${ }^{*} P<0.05,{ }^{* *} P<0.01$, ${ }^{* * *} P<0.001$. of tubular epithelial cells (Supplemental Figure 1A) $(15,16,24)$. In the absence of the ROSA26-ACTB-tdTomato,-EGFP reporter allele, EGFP was not detectable in kidneys from 8-week-old mice, confirming that the Foxd1 promoter is no longer active in adults (Foxd1 ${ }^{\mathrm{Cr} / \mathrm{+}}$ mice express an EGFP/Cre fusion protein during development; Supplemental Figure 5A).

We next examined to what degree cortical and outer medullary FOXD1 stroma-derived interstitial cells contributed to EPO production in Foxd1-Phd $2^{-/-}$kidneys by using multiplex ISH using fluorescent probes against EGFP and Epo transcripts. We found that the number of cells that actively transcribed Epo increased from $2.5 \pm 1.7$ cells $/ \mathrm{mm}^{2}$ in 8 -week-old Foxd1- $\mathrm{mT} / \mathrm{mG}$ control mice to $117.4 \pm 25.7$ cells $/ \mathrm{mm}^{2}$ in age-matched Foxd1-mT/mG-Phd2mutants ( $n=5$ each; $P<0.05$; Figure 4 ). This large change in the number of interstitial cells that transcribe Epo was furthermore reflected in the increased representation of $\mathrm{Epo}^{+}$cells among $E G F P^{+}$interstitial cells $\left(39.0 \% \pm 5.5 \%\right.$ of $E G F P^{+}$interstitial cells were $E p o^{+}$vs. $0.8 \% \pm 0.5 \%$ in controls; $\left.P<0.001\right)$ and correlated with an increase in Epo mRNA levels measured by real-time PCR in whole-kidney homogenates. We then compared REPC numbers in Foxd1-mT/mG-Phd2 ${ }^{-/}$versus Foxd1- $m T / m G$ control mice that were wild type for $P h d 2$ and were made anemic by phlebotomy (average Hct of $17.8 \% \pm 0.8 \%$ ). In anemic control mice the number of REPCs increased to $221.9 \pm 38.0$ cells $/ \mathrm{mm}^{2}$ compared with 117.4 \pm 25.7 cells $/ \mathrm{mm}^{2}$ in Foxd1-mT/mG-Phd2 ${ }^{--}$mutants, suggesting that Epo induction in Foxd1-mT/mG-Phd2-/- kidneys was submaximal and did not reach full potential (Figure 4 ).

We next sought to investigate whether the pool of cells that transcribed Epo in Foxd1-Phd2-- kidneys was expandable, and asked whether the provision of additional hypoxic stimuli would lead to a further increase in EPO production. We phlebotomized Foxd1-mT/mG-Phd2 $2^{--}$mice to an average Hct of $35.8 \% \pm 4.3 \%$ and found that phlebotomy further increased REPC numbers and total renal Epo levels $\left(365.5 \pm 25.9\right.$ cells $/ \mathrm{mm}^{2}$ vs. $117.4 \pm 25.7$ cells $/ \mathrm{mm}^{2}$; 


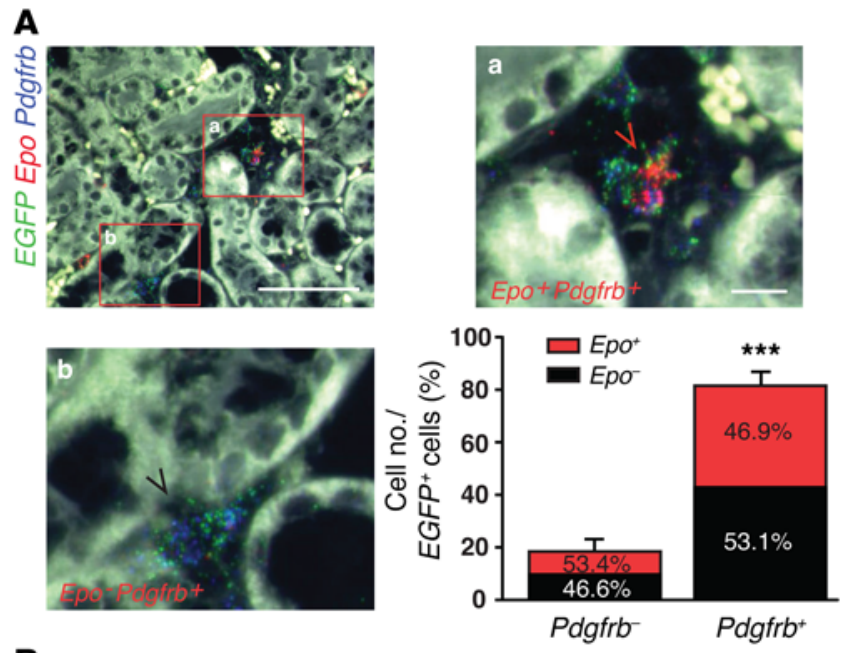

B

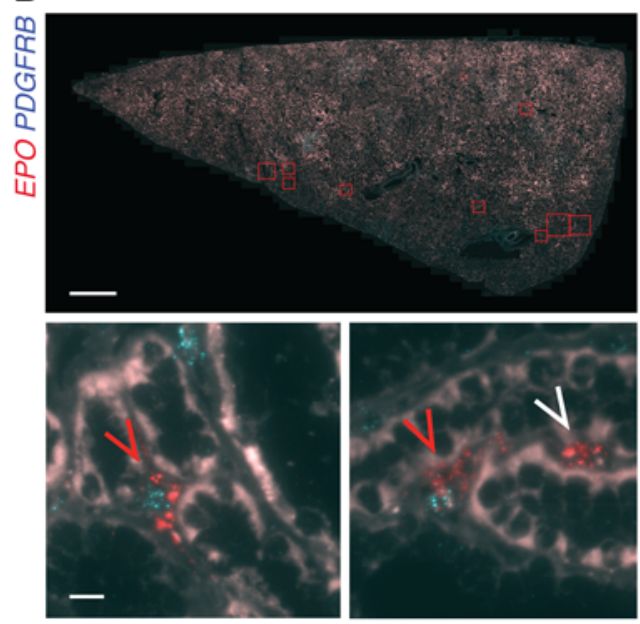

$73.0 \% \pm 4.1 \%$ of $E G F P^{+}$interstitial cells were $E p o^{+}$in phlebotomized vs. $39.0 \% \pm 5.5 \%$ in nonphlebotomized mutants; $n=5$ each; $P<$ 0.001; 3.5-fold increase in Epo levels; $P<0.05$; Figure 4). Interestingly, we also observed a significant increase in the absolute number of $E G F P^{+}$interstitial cells in kidneys from $P h d 2^{---}$mice subjected to phlebotomy (Figure 4). Taken together our data indicate that $P h d 2$ inactivation in FOXD1 stroma-derived interstitial cells resulted in submaximal EPO production, which was associated with Epo induction in only a subset of $P h d 2$-deficient renal interstitial cells. However, $P h d 2$-deficient renal interstitial cells that failed to express Epo retained EPO-producing capacity and converted to Epo-expressing cells upon provision of a hypoxic stimulus.

Different subpopulations of renal interstitial cells give rise to REPCs. To further characterize Phd2-deficient interstitial cells that failed to induce Epo, we analyzed the expression of Pdgfrb, a cellular marker of REPCs, pericytes, and other fibroblast-like perivascular cells. Since only $50 \%$ of EPO-producing cells were reported to express PDGFRB using an EPO reporter transgenic line (25), we hypothesized that $P h d 2^{--} E p o^{-}$cells may lack PDGFRB expression. Multiplex ISH using probes against EGFP, Epo, and Pdgfrb, however, demonstrated that while the majority of $E p o^{+}$cells expressed $P d g f r b, E p o^{+}$cells were not differently distributed between Pdgfr $b^{+}$ and Pdgfrb cell populations $(46.9 \% \pm 3.9 \%$ vs. $53.4 \% \pm 10.1 \%$, respectively; Figure $5 \mathrm{~A})$. Our findings were consistent with real-
Figure 5. Differential expression of PDGFRB in murine and human EPOproducing renal interstitial cells. (A) Shown are representative images from ISH studies simultaneously detecting Epo (red signal), EGFP (green signal), and Pdgfrb (blue signal) transcripts in formalin-fixed, paraffinembedded kidney tissue sections from Foxd1-Phd2-/- mutant mice. The red arrowhead depicts a representative $E p o^{+} P d f g r b^{+}$interstitial cell; the black arrowhead depicts a representative Epo-Pdgfrb cell. Bottom right panel: Quantification of Epo-expressing cells within Pdgfrb and Pdgfrbcell populations expressed as percentage $(n=4)$. Scale bars: $50 \mu \mathrm{m}$ (lowmagnification panel), $10 \mu \mathrm{m}$ (high-magnification panels). Graph bars represent mean \pm SEM; 2 -tailed Student's $t$ test; ${ }^{* *} P<0.001$. (B) ISH for EPO (red signal) and PDGFRB (blue signal) in human renal tissue. Top panel: Low-magnification image of large tissue section from normal kidney. $E P O$-expressing cells were detected in areas highlighted by red boxes (total of $63 \mathrm{EPO}^{+}$cells; $12 \mathrm{EPO}^{+}$cells were negative for $P D G F R B$ ). Bottom panels: Representative images of cells expressing both PDGFRB and EPO (red arrowheads) and interstitial EPO-expressing cells with nondetectable PDGFRB transcripts (white arrowhead). Scale bars: $1 \mathrm{~mm}$ (top panel), $10 \mu \mathrm{m}$ (bottom panels).

time PCR data obtained from single-cell isolates enriched for PDGFRB-expressing cells using magnetic beads, which indicated that the majority of Epo transcripts were contained within the $\mathrm{PDGFRB}^{+}$cell fraction (Supplemental Figure 5B). With regard to cellular identity and spatial distribution of $E p o^{+} P d g f r b^{-}$cells, $E p o^{+}$ cells were not found to express transcripts encoding $\alpha$-smooth muscle actin (ACTA2) and were never associated with vessel walls (Supplemental Figure 5C). To further examine REPC heterogeneity with regard to PDGFRB expression, we analyzed the expression of EPO and PDGFRB in human nephrectomy specimen and renal biopsy material. Most EPO-expressing renal interstitial cells localized to the inner cortex and were organized in clusters (Figure 5B). We detected $E P O$-expressing cells in 4 of 9 nephrectomies and 2 of 5 routine renal biopsy cores. Due to the low yield of EPOexpressing cells in renal biopsy cores, which is most likely due to their small size and to limitations in biopsy depth, we focused our analysis on the larger nephrectomy specimen. Despite the variability in the total number of EPO-expressing cells (4-63 EPO cells per section) and the number of cells that were $E P O^{+} P D G F R B^{-}$ (0-12 cells per section), these findings in human tissue support our observations in mice that PDGFRB is differentially expressed in REPCs. Taken together our data suggest that renal interstitial cells, which fail to induce Epo in response to Phd2 inactivation, cannot be distinguished based on Pdgfrb expression.

To further define the cellular heterogeneity of $P h d 2^{-/-}$renal interstitial cells, we examined EPO responses in a specific subpopulation of pericytes and perivascular fibroblasts using a Cre-recombinase transgene under the control of the chondroitin sulfate proteoglycan 4 (Cspg4) promoter. CSPG4 is also known as neuro-glial antigen 2 (NG2) and is expressed in a subpopulation of renal pericytes $(26,27)$. We generated $\mathrm{Ng} 2$-Cre Phd $2^{f / f l}$ mice, from here on referred to as $\mathrm{Ng} 2-\mathrm{Phd} 2^{--}$mutants, and examined whether these mice responded to $P h d 2$ inactivation with increased renal Epo transcription. Surprisingly, $\mathrm{Ng} 2-\mathrm{Phd} 2^{-/-}$mice were characterized by normal renal Epo transcript levels and did not develop polycythemia. Furthermore, renal Epo transcription was equally suppressible in $\mathrm{Ng} 2-\mathrm{Phd2} \mathrm{2}^{-/}$and control mice following the administration of recombinant human EPO, which resulted in the development of polycythemia. This finding suggested 

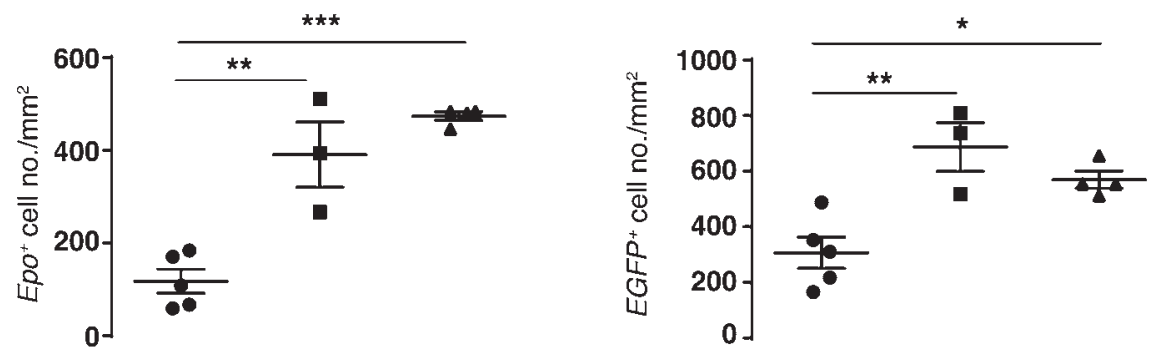

Figure 6. Inactivation of Phd1 and Phd3 increases renal EPO production in Phd2-/- kidneys. Shown are the results from multiplex fluorescent ISH studies using formalin-fixed, paraffin-embedded kidney tissue sections from Foxd1-mT/mG-Phd2 $2^{--}\left(\right.$Phd2 $\left.^{-1-}\right)$ mutants $(n=5)$,

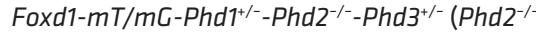
Phd1 $1^{+-}$Phd $\left.3^{+-}\right)$mutants $(n=3)$ and Foxd1-mT/ mG-Phd2-/- mice treated with GSK1002083A (Phd2 $\left.2^{--}+\mathrm{PHI} ; n=4\right)$. Top left panel: Quantification of Epo-expressing cells. Shown is the absolute number of $\mathrm{Epo}^{+}$cells per square millimeter. Bottom left panel: Epo mRNA levels relative to 185 in total kidney tissue homogenates as determined by real-time PCR $(n=3-6)$. Top right panel: Quantification of EGFP-expressing cells. Shown is the absolute number of $E G F P^{+}$cells per square millimeter of kidney tissue. Bottom right panel: Fraction of Epo-expressing cells within the $\mathrm{ECFP}^{+}$cell population. Data are represented as mean $\pm \mathrm{SEM}$; 1-way ANOVA with post hoc Tukey's test; ${ }^{*} P<0.05,{ }^{* *} P<0.01,{ }^{* *} P<0.001$.

that $\mathrm{Ng} 2-\mathrm{Ph} \mathrm{d}^{-/-}$mice behaved physiologically normally and that Epo transcription was not constitutively active as a result of $P h d 2$ inactivation (Supplemental Figure 6). In contrast to Phd2 inactivation, phlebotomy induced Epo in cells labeled with $\mathrm{Ng2-Cre}$ (Supplemental Figure 6). These data indicate that renal interstitial cells with a history of $\mathrm{Ng} 2$-Cre expression behaved similarly to FOXD1 stroma-derived cells that failed to express Epo in Foxd1-Phd2 ${ }^{-/-}$kidneys, and furthermore raise the possibility that NG2 expression may be associated with renal interstitial cells that differ in their responsiveness to $P h d 2$ inactivation and thus regulation of HIF-2 activity and EPO production.

Differential regulation of HIF-2 activity in FOXD1 stroma-derived renal interstitial cells. Although inactivation of Phd1 and/or Phd3 by itself does not induce renal Epo (Supplemental Figure 3), it is not known to what degree PHD1 and/or PHD3 have a modulatory effect on EPO responses in the kidney. Because we found that inactivation of Phd2 induced Epo transcription in only a limited number of FOXD1 stroma-derived interstitial cells, we asked whether additional pharmacologic inhibition of PHD catalytic activity had any further effect on REPC pool size and/or renal EPO output in Foxd1-Phd $2^{--}$mutant mice. To examine this, we treated Foxd1$m T / m G-P h d 2^{--}$mice with GSK1002083A, a PHI, which reversibly inhibits the catalytic activity of all PHDs (3). Treatment with PHI raised the number of cells that transcribed Epo from $117.4 \pm 25.8$ to $473.9 \pm 9.26$ cells $/ \mathrm{mm}^{2}$ ( $n=5$ and 4 , respectively; $P<0.001$ ), which correlated with an approximately 12 -fold increase in renal Epo mRNA, and suggested that the additional inhibition of PHD1 and/or PHD3 catalytic activity was required for the conversion of non-Epo-expressing $P h d 2^{-/}$interstitial cells to cells that transcribe Epo $\left(83.6 \% \pm 3.3 \%\right.$ of $E G F P^{+}$interstitial cells were $E p o^{+}$; Figure 6). The increase in REPC numbers in PHI-treated Foxd1-Phd $2^{-/}$mice was associated with an increase in the number HIF-2 $\alpha$-expressing interstitial cells (Supplemental Figure 4); HIF-2 is solely responsible for the induction of Epo in Phd $2^{-/-}$kidneys, as demonstrated by genetic means in mice that were double-deficient for Phd2 and Hif2a (Supplemental Figure 7).
To further investigate these findings we used a genetic approach and found that the combined heterozygous deletion of Phd1 and $P h d 3$ in $P h d 2^{-/-}$kidneys (Foxd1-mT/mG-Phd1 ${ }^{+/-}-P h d 2^{-/-}-P h d 3^{+/-}$) was sufficient to significantly increase REPC number from $117.4 \pm 25.8$ to $390.8 \pm 70.57$ cells $/ \mathrm{mm}^{2}$ ( $n=5$ and 3 , respectively, $P<0.01$ ), which was reflected in an approximately 4.5 -fold increase in renal Epo mRNA levels ( $n=3$ and 6, respectively; Figure 6). To further assess the relative contributions of PHD1 and PHD3 to the regulation of REPC pool size in Foxd1-Phd2 ${ }^{-/-}$mutants, we generated Foxd1-Phd1 ${ }^{-/-}$

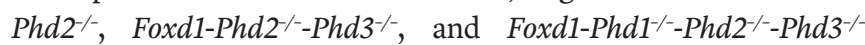
triple mutant mice. Whereas Foxd1-Phd2 ${ }^{--}$mutants were born in mendelian ratios and developed normally, homozygous inactivation of all 3 Phd alleles or the combined inactivation of Phd 2 and Phd3 resulted in juvenile lethality, precluding the analysis of REPC pool size and renal Epo levels in adults that either lacked all copies of $P h d 1$, $P h d 2$, and $P h d 3$ or were double-deficient for Phd2 and Phd3 (data not shown). In contrast, dual targeting of $P h d 1$ and $P h d 2$ resulted in normal development and did not further increase renal Epo mRNA levels compared with $P h d 2$ inactivation alone (Supplemental Figure 8), suggesting that (a) PHD1 is unlikely to play an adjunctive role in regulating REPC pool size in $P h d 2^{--}$kidneys and (b) inhibition of PHD3 activity was most likely responsible for the additional increase

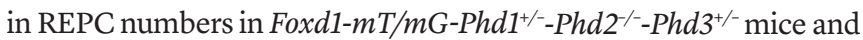
PHI-treated Foxd1-Phd2-- mutants. However, since we were unable to assess renal EPO production in adult $P h d 2 / 3$ double and $P h d 1 / 2 / 3$ triple mutants, we cannot exclude with certainty that PHD1 plays a contributory role in the regulation of REPC pool size.

In anemic mice the increase in REPC numbers is usually associated with their spatial expansion toward the outer cortex (Figure 7). To investigate whether the topography of REPCs in Foxd1-Phd2-mutants followed a specific pattern or whether it was similar to that found in anemic mice, we examined the spatial distribution of $\mathrm{EpO}^{+}$ interstitial cells in the outer, mid-, and inner renal cortex (Figure 7). In $P h d 2^{-/-}$or $P h d 1^{+/-}-P h d 2^{-/-}-P h d 3^{+/-}$knockout mice REPCs were distributed equally throughout the entire cortex, whereas the majority of $E p O^{+}$cells in anemic control mice localized to the inner cortex (Fig- 

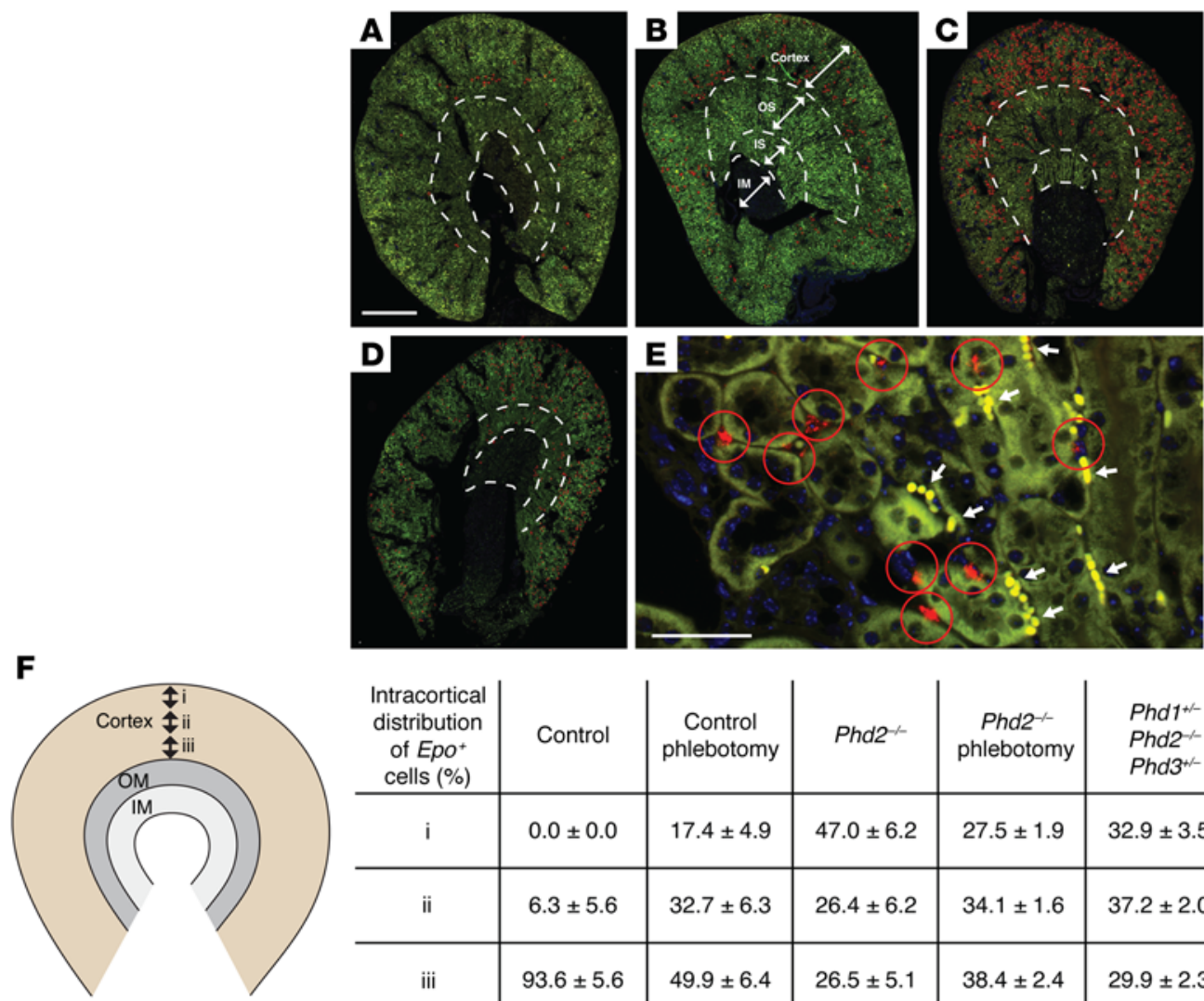

\begin{tabular}{|c|c|c|c|c|c|c|}
\hline $\begin{array}{c}\text { Intracortical } \\
\text { distribution } \\
\text { of } E p o^{+} \\
\text {cells }(\%)\end{array}$ & Control & $\begin{array}{c}\text { Control } \\
\text { phlebotomy }\end{array}$ & Phd2 & $\begin{array}{c}\text { Phd2- } \\
\text { phlebotomy }\end{array}$ & $\begin{array}{l}\text { Phd1+ } \\
\text { Phd2- } \\
\text { Phd } 3^{+-}\end{array}$ & $\begin{array}{l}\mathrm{Phd2}^{-} \\
+\mathrm{PHI}\end{array}$ \\
\hline i & $0.0 \pm 0.0$ & $17.4 \pm 4.9$ & $47.0 \pm 6.2$ & $27.5 \pm 1.9$ & $32.9 \pm 3.5$ & $31.2 \pm 3.5$ \\
\hline ii & $6.3 \pm 5.6$ & $32.7 \pm 6.3$ & $26.4 \pm 6.2$ & $34.1 \pm 1.6$ & $37.2 \pm 2.0$ & $33.3 \pm 1.7$ \\
\hline iii & $93.6 \pm 5.6$ & $49.9 \pm 6.4$ & $26.5 \pm 5.1$ & $38.4 \pm 2.4$ & $29.9 \pm 2.3$ & $29.9 \pm 6.0$ \\
\hline
\end{tabular}

Figure 7. REPC topography in anemic mice differs from that in Foxd1-Phd2-/- mice. Shown are representative annotated images illustrating the distribution of REPCs in anemic control and in Foxd1-Phd2 ${ }^{--}$mice. Epo ISH studies were performed using formalin-fixed, paraffin-embedded kidney tissue sections. (A-D) Epo transcripts were detected by red signals in original images. To facilitate visualization and to provide an overview of Epo transcript distribution at low magnification, Epo+ cells were annotated with red circles. (A) Kidney section from control mouse at baseline. (B) Kidney section from control mouse with Hct of $26 \%$. (C) Kidney section from control mouse with Hct of $15 \%$. (D) Representative low-magnification image of kidney from a Foxd1-Phd2 ${ }^{-1-}$ mutant. (E) Representative high-magnification image of Epo ISH from control mouse with Hct of $15 \%$ to illustrate how Epo+ cells were annotated. rbc are denoted by white arrows. White dashed lines indicate borders between cortex, outer stripe (OS) and inner stripe (IS) of outer medulla, and inner medulla (IM). Scale bars: $1 \mathrm{~mm}(\mathbf{A}-\mathbf{D}), 50 \mu \mathrm{m}(\mathbf{E})$. (F) Shown is the relative distribution of Epo-expressing cells in the outer (i), mid- (ii), and inner (iii) renal cortex from control mice at baseline $(n=4)$ and after phlebotomy $(n=5)$, Foxd1-mT/mG-Phd2 $2^{-/-}\left(P h d 2^{-/-}\right)$mutants at baseline $(n=5)$ and after phlebotomy $(n=5)$, Foxd1-mT/mG-

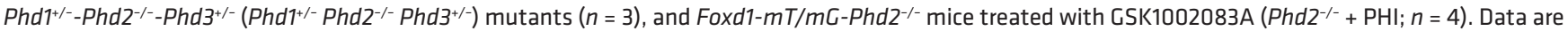
represented as mean \pm SEM. OM, outer medulla.

ure 7). These findings suggest that the distribution of renal interstitial cells that respond to $P h d$ inactivation with Epo induction does not follow an oxygen gradient and that their responsiveness to genetic Phd inactivation may be independent of tissue oxygen levels.

Differential expression of Phd1 and Phd3 in Foxd1-Phd2mutants. Our genetic studies suggested that HIF-2 $\alpha$ stabilization and thus induction of Epo transcription were prevented by PHD1/3-mediated HIF prolyl-4-hydroxylation in a subpopulation of $\mathrm{Phd}^{--}$interstitial cells. We therefore hypothesized that $\mathrm{Epo}^{+} \mathrm{Phd}^{-/}$and $\mathrm{Epo}^{-} \mathrm{Phd} 2^{--}$renal interstitial cells differed with regard to $P h d 1$ and $P h d 3$ expression and that the fraction of Epo $P h d 2^{-\digamma}$ cells that expressed $P h d 1$ and/or Phd 3 might be increased compared with that of $\mathrm{EPO}^{+} \mathrm{Phd} 2^{--}$cells. To test this hypothesis we performed multiplex ISH with probes against EGFP, Epo, and either $P h d 1$ or $P h d 3$ transcripts. We found that the majority of Epoexpressing $P h d 2^{--}$cells did not express detectable levels of Phd1

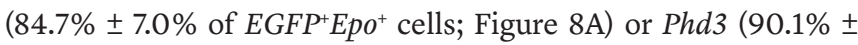
$2.2 \%$ of $E G F P^{+} E p o^{+}$cells; Figure $\left.8 \mathrm{~A}\right)$. In contrast, a larger proportion of cortical and outer medullary $E G F P^{\dagger} E p o^{-}$cells expressed
Phd1 $(42.7 \% \pm 13.8 \%)$ or Phd3 $(23.5 \% \pm 5.5 \%)$, suggesting (a) the possibility that the presence of PHD1 and/or PHD3 prevented HIF-2 activation in Phd2 ${ }^{-}$cells that failed to express Epo, and (b) that inactivation of PHD1 and PHD3 by genetic or pharmacologic (PHI treatment) means was required for the conversion of these cells to EPO-producing interstitial cells (Figure 8B). For technical reasons we were unable to simultaneously hybridize against $P h d 1$, $P h d 3, E p o$, and EGFP, and could therefore not directly determine to what degree Phd1- and Phd3-expressing EPo- subpopulations overlapped. However, we estimate from separate ISH studies that up to $45 \%$ of either Phd1- or Phd3-expressing cells can express both genes (data not shown). In summary, our data suggest that differences in Phd1 and Phd 3 expression modulate HIF-2 and EPO responses in FOXD1 stroma-derived renal interstitial cells.

\section{Discussion}

Here we used a genetic approach to investigate the role of the 3 HIF-prolyl-4-hydroxylases, PHD1, PHD2, and PHD3, in the regulation of REPC plasticity and renal EPO production. We 
A
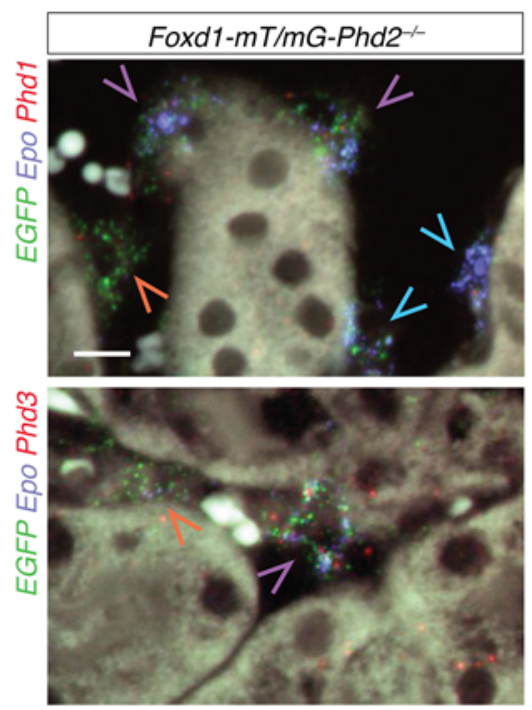
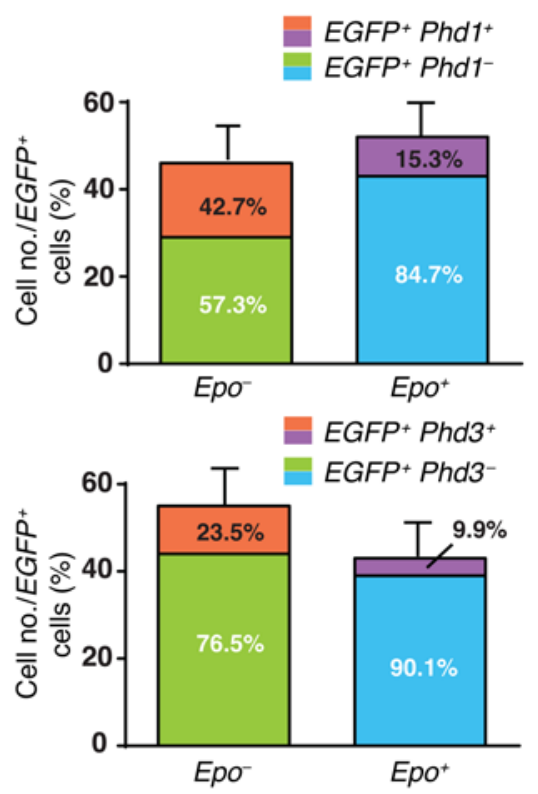

B

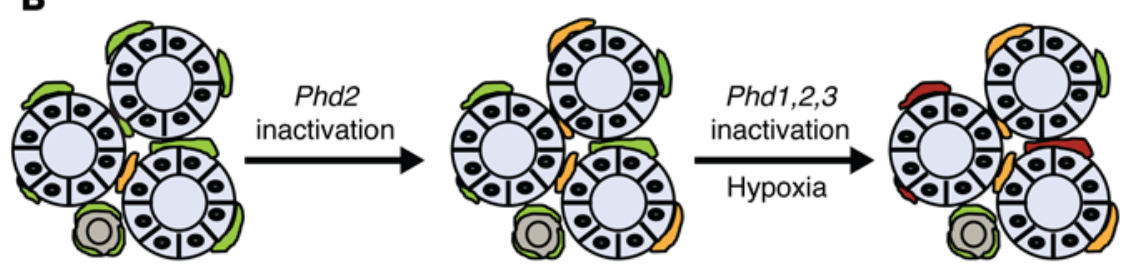

Figure 8. Phd1 and Phd3 are differentially expressed in Phd $^{-/-}$renal interstitial cells. (A) Results from multiplex fluorescent ISH studies using formalin-fixed, paraffin-embedded kidney tissue sections from Foxd1-mT/mG-Phd2-/- mice detecting Epo (blue signal), EGFP (green signal), and Phd1 or Phd3 transcripts (red signal). Shown are representative images; blue arrowheads depict $E G F P^{+} E \mathrm{Po}^{+} P h d 1^{-}$cells, purple arrowheads depict EGFP+E ${ }^{+} \mathrm{O}^{+} \mathrm{Phd1} 1^{+}$or EGFP+Epo+Phd3 $3^{+}$cells, and orange arrowheads point toward EGFP ${ }^{+} E p o^{-} P h d 1^{+}$or EGFP+Epo-Phd3 ${ }^{+}$cells. Scale bar: $10 \mu \mathrm{m}$. Quantification of cells is shown in the right panels ( $n=3$ each group). Data are represented as mean \pm SEM. (B) Schematic depicting the role of individual PHDs in the regulation of REPC number and renal EPO output. EPO-producing cells are indicated by orange and burgundy red color. Renal interstitial cells that do not produce EPO are indicated by green. Renal tubules are depicted by gray. demonstrate that cells with EPO-producing capacity are derived from FOXD1-expressing stromal progenitor cells and identify a subpopulation of renal interstitial cells that respond to $P h d 2$ inactivation with the induction of Epo. We also provide evidence that PHD3 plays an adjunctive role in the regulation of plasma EPO levels, as it modulates the number of renal interstitial cells that respond to Phd2 inactivation with EPO synthesis. We propose that EPO-producing renal interstitial cells derive from distinct subpopulation of FOXD1 lineage cells, which vary in their ability to regulate HIF-2 $\alpha$ degradation, partly as a result of differences in PHD activity levels.

Renal peritubular interstitial cells begin to synthesize EPO when HIF- $2 \alpha$ is stabilized, either under hypoxic conditions or as a result of HIF-PHD inhibition by pharmacologic or genetic means (1). Here we demonstrate by cell-specific gene targeting that cells with EPO-producing capacity are entirely contained within FOXD1 lineage-derived interstitial cells. However, a recent study reported that cells with EPO-producing ability are partly derived from PO lineage cells, which overlap with FOXD1-expressing progenitor cells that start to surround the cap mesenchyme from E10.5 onward $(11,14)$. While the exact interrelationships between those two cell types are not known, it has been suggested that neural crest-derived cells migrate into the developing kidney at E13.5, start to express FOXD1, and replenish existing FOXD1 progenitor cells $(11,14)$. Since previous studies were not able to assign all EPO-producing cells to the PO lineage (14), it is plausible that non-PO lineage interstitial cells with EPO-producing capacity are derived from FOXD1 stromal cells that existed prior to E13.5.

PDGFRB is a well-recognized marker of both pericytes and REPCs, and recent imaging studies support the notion that renal pericytes are capable of synthesizing EPO (28). In our genetic model of Foxd1-Cre-mediated Phd2 inactivation we detected $P d g f r b$ transcripts in approximately $80 \%$ of Epo-expressing interstitial cells, suggesting that PDGFRB may not be an exclusive marker of REPCs. Since we did not find that $E \mathrm{PO}^{+}$cells expressed Acta 2 or were associated with vessel walls in any of our studies, it is unlikely that $E p o^{+} P d g f r b^{-}$cells represent VSMCs. Although our studies with Pdgfrb-Cre Phd $2^{f / f l}$ mice suggested that the sensitivity of ISH detecting Pdgfrb in REPCs is greater than 90\% (Supplemental Figure 9), it is possible that Pdgfrb transcript levels in these cells were below the detection threshold of ISH. Nevertheless, further studies are warranted to investigate the possibility of functional differences between $E p o^{+} P d g f r b^{+}$cells and cells that we identified as $E p o^{+} P d g f r b^{-}$using ISH.

A clinical hallmark of advanced CKD is anemia, which, although multifactorial, is primarily attributable to relative EPO deficiency (1). As a result of renal injury, renal interstitial cells lose their ability to respond to hypoxia with adequate and sufficient EPO production resulting in anemia. Whether this phenomenon is due to a change in the regulation of HIF- $2 \alpha$ production and/ or degradation or results from impaired EPO transcription in the 
presence of nuclear HIF-2 is unclear. However, increased NF- $\kappa$ B signaling and transdifferentiation into myofibroblasts have been suggested as potential underlying mechanisms for the development of renal anemia $(14,29)$. The reduced ability to mount an adequate EPO response appears to be reversible to a certain degree, as treatment with glucocorticoids or removal of fibrotic stimuli in experimental fibrosis models protected the EPO-producing ability of the injured kidney $(14,29)$. In a recent genetic study in mice, Souma and colleagues showed that PHD inactivation in Epo-expressing cells was capable of reversing the decline of renal EPO production in a unilateral ureteral obstruction model (28). This study made use of a Cre-recombinase transgene that was controlled by Epo regulatory elements and thus was oxygenregulated, i.e., inducible by hypoxia. $P h d 2$ inactivation alone or in conjunction with either Phd1 and/or Phd3 using Epo-Cre resulted in the development of polycythemia. Inactivation of Phd1, Phd2, and Phd3 was associated with renal Epo transcript levels that were similar to control, which is in contrast to our study, where large increases in Epo mRNA were detected with Phd2 inactivation alone. This discrepancy is explained by the very small number of renal interstitial cells targeted with the use of the Epo-Cre transgene. The ability of PHD inhibition to protect the kidney's EPOproducing ability under injury conditions suggests that pharmacologic HIF activation increases EPO synthesis in fibrotic kidneys either by converting non-EPO-producing renal interstitial cells into REPCs, or, as proposed by Souma and colleagues, by maintaining Epo transcription in EPO-producing cells that are undergoing myofibroblast transformation (28). The possibility that even severely diseased kidneys do not completely lose their ability to synthesize EPO in response to HIF-2 activation has been suggested based on studies in CKD patients whose serum EPO levels increased upon ascent to high altitude or following treatment with HIF-2 $\alpha$-stabilizing compounds (30-32). While the tissue source of EPO was not determined in these studies, this notion is furthermore supported by a large retrospective clinical study, which showed that dialysis patients living at high altitude required lower doses of recombinant EPO to maintain $\mathrm{Hb}$ (33).

While recombinant forms of EPO have been a mainstay of renal anemia therapy for almost 3 decades, cardiovascular safety concerns have been raised regarding the use of recombinant EPO and prompted the FDA to issue a black-box warning $(34,35)$. Although the underlying molecular mechanisms for the increase in adverse cardiovascular events are still unclear, they may be related to the repeated administration of recombinant EPO doses that generate very high and supraphysiologic plasma EPO levels (36). Oral therapy with HIF- $\alpha$-stabilizing PHD-inhibiting compounds is currently being investigated in phase III clinical trials, and has the potential to provide a more physiologic and potentially safer therapeutic approach to renal anemia (1). Our data would argue that renal interstitial cells with EPO-producing potential exhibit differential sensitivity to HIF-prolyl-4-hydroxylase inhibition, especially if compounds that target PHDs with different efficacy are used. Our data furthermore support the concept that compounds that specifically inhibit PHD2 are very effective in stimulating renal EPO synthesis. The efficacy of such PHD2-specific compounds could be enhanced or modulated by hypoxia, as phlebotomy substantially increased the number of Epo-transcribing cells in $\mathrm{Phd2^{-/- }}$ kidneys.
Although renal interstitial fibroblast-like cells/pericytes and hepatocytes are the main cellular sources of EPO under most physiologic conditions (1), other cell types, such as osteoblasts and astrocytes, have been shown to synthesize EPO when HIF was activated by genetic means in mice $(37,38)$. Therefore these cell types have potential to contribute to plasma EPO upon systemic administration of PHIs. In the kidney, genetic studies showed that HIF-2 activation via $V h l$ gene deletion converted renin-producing cells to EPO-producing cells $(39,40)$. Whether these cells are also capable of synthesizing EPO in response to PHD2 inactivation is currently not known.

While renal Epo mRNA levels correlated with REPC pool size in our study, we also observed a statistically significant rise in the absolute number of EGFP-labeled FOXD1 stroma-derived interstitial cells when $\mathrm{Phd2^{-- }}$ mice were phlebotomized or PHI-treated, suggesting a physiologic response rather than a developmental abnormality. To what degree this increase in EGFP-labeled cells was transient, or whether it had any adverse consequences on renal function, is currently being investigated in our laboratory. While it is plausible that sustained HIF activation during nephrogenesis affects interstitial cell numbers in the adult kidney, an association between interstitial cell proliferation and increased EPO production has recently been described in rats treated with poly-D-glutamic acid (41); furthermore, hypoxia has been shown to stimulate pericyte proliferation in vitro (42). From genetic fate-tracing studies, it has become evident that FOXD1 stroma-derived interstitial cells and pericytes have the potential to proliferate. While the underlying molecular mechanisms are complex and involve multiple signaling molecules, FOXD1 stroma-derived interstitial cells proliferate and transdifferentiate into myofibroblasts under injury conditions, which leads to excessive extracellular matrix production and the development of fibrosis $(11,15)$.

While HIF-2 is the main regulator of EPO in kidney and liver $(3,43)$, renal interstitial cells and hepatocytes differ from each other with regard to PHD-mediated control of HIF-2 $\alpha$ degradation. While our study identified at least 2 interstitial cell populations in the kidney that displayed differential sensitivity to $P h d 2$ inactivation, inactivation of at least 2 HIF-PHDs is required for moderate Epo induction in hepatocytes. To achieve very high levels of Epo induction in the liver, inactivation of all 3 HIF-PHDs is required (21-23). The role of individual PHDs in the regulation of EPO in other cell types, however, is not clear. Inactivation of all 3 HIFPHDs appeared to be necessary for the induction of Epo in osteoblasts (38), while Phd2 deletion alone activated Epo transcription in neurons (44). In our study, PHD3, and not PHD1, was critical for the regulation of REPC pool size in the absence of PHD2. PHD3 itself is HIF-regulated and has been shown to be part of a negative-feedback loop that suppresses HIF activity under hypoxia and reoxygenation conditions $(45,46)$. Thus it is plausible that interstitial cells that respond to Phd2 inactivation with EPO synthesis are more sensitive to oxygen deprivation and may represent a distinct population of renal interstitial cells that is part of a first-line defense against hypoxia. Our data suggest that failure to activate Epo in $\mathrm{Phd} 2^{-/-}$cells does not reflect increased availability of oxygen; the presence of oxygen is predicted to keep PHD1 and PHD3 catalytically active and thus suppress HIF-2 activation, i.e., Epo induction. If this were the case, one would predict that the spa- 
tial distribution of Epo-expressing cells followed the intracortical oxygen gradient (47) and would be similar to the distribution of REPCs under hypoxic conditions. However, this was not observed in our studies. Whether cells that respond to Phd2 inactivation with EPO synthesis are functionally distinct in other respects remains to be investigated.

In summary, we have shown that renal interstitial cells with EPO-producing capacity are derived from FOXD1-expressing stromal progenitors and are heterogeneous with regard to their responsiveness to $P h d 2$ inactivation, regulation of HIF-2 activity, and EPO production. Our genetic data identify PHD2 as a main, and PHD3 as an adjunctive, regulator of REPC plasticity. These findings have immediate clinical relevance as HIF-PHIs are currently being evaluated in large clinical trials for the treatment of renal anemia. To what degree different subpopulations of renal interstitial cells with EPO-producing capacity differ in their sensitivity to renal injury and contribution to the pathogenesis of renal anemia warrants further investigation.

\section{Methods}

Generation and genotyping of mice and animal procedures. The generation and genotyping of mice with floxed alleles for Epo, Hif2a (Epas1), Phd1 (Egln2), Phd2 (Egln1), and Phd3 (Egln3) have been described previously $(18,20,48-50)$. To target FOXD1 stroma-derived cells, floxed alleles were bred to mice that expressed Foxd1-Cre (16). Foxd1 ${ }^{\text {Cre/+ }}$

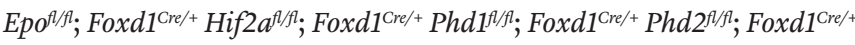

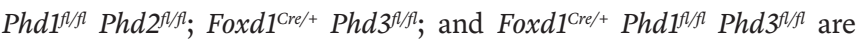
referred to as Foxd1-Epo-/, Foxd1-Hif2a-/, Foxd1-Phd1-/, Foxd1-Phd2--, Foxd1-Phd1 1/-Phd2 ${ }^{-/-}$, Foxd1-Phd3 ${ }^{-/-}$, and Foxd1-Phd1 ${ }^{-/-}$-Phd3 $3^{-/}$, respectively. For the inactivation of $P h d 2$ alone we initially used 2 different mouse lines, which produced identical results $(20,48)$. For the inactivation of multiple Phd alleles and for ISH studies, the floxed Phd2 allele described in ref. 48 was used. To visualize cells that had undergone Foxd1-Cre-mediated recombination, mice were bred to mice that carried the ROSA26-ACTB-tdTomato,-EGFP double-fluorescent Crereporter strain, generating Foxd1-mT/mG mice (17). For the targeting of Phd2 in PDGFRB-expressing and NG2-expressing renal interstitial cells, Pdgfrb-Cre and Cspg4-Cre transgenic mice were used (51, 52). Anemia was induced by retro-orbital phlebotomy, and hypoxia experiments were performed in an animal hypoxia chamber (BioSpherix) as previously described (3).

Analysis of blood samples and renal interstitial cell isolation. Hct, $\mathrm{rbc}, \mathrm{Hb}$, serum EPO level, and reticulocyte counts were determined as described previously (50). For the isolation of renal PDGFRB-expressing interstitial cells, kidneys were digested as previously described (53), and cells were purified by immunomagnetic bead separation using antibodies directed against PDGFRB (catalog 14-1402-82; eBioscience) and anti-rat IgG microbeads (catalog 130-048-502; Miltenyi Biotec).

Treatment with prolyl-4-hydroxylase inhibitor (PHI) and recombinant human EPO. For pharmacologic HIF activation, the prolyl-4hydroxylase inhibitor GSK1002083A (GlaxoSmithKline) was dissolved in $1 \%$ methylcellulose and administered by oral gavage at a dose of $60 \mathrm{mg} / \mathrm{kg}$ at 24 and 4 hours before tissue harvest (3). Recombinant human EPO (Amgen) was dissolved in $0.1 \mathrm{ml}$ water and injected i.p. at a dose of 200 IU every other day for a period of 10 days.

$D N A, R N A$, and protein analysis. RNA was isolated using the RNeasy kit according to the manufacturer's protocol (Qiagen). For quantitative real-time PCR, cDNA was prepared from $1 \mu \mathrm{g}$ of total RNA and analyzed using SYBR green or TaqMan PCR Master Mix on an ABI 7300 or StepOnePlus platform (Applied Biosystems). Primer sequences used for the detection of Epo, Vegf, and Phd3 have been described previously $(3,50) .18 S$ rRNA was used to normalize mRNA. For the quantification of mRNA expression levels, the relative standard curve method was used according to the manufacturer's instructions (Applied Biosystems); standard curves were generated for each real-time PCR run, and for each figure panel relative mRNA expression levels are derived from a different standard curve. Western blot analysis was performed with nuclear protein extracts as previously described (43) using anti-HIF-1 $\alpha$ (catalog 10006421; Cayman Chemical Co.) and anti-HIF-2 $\alpha$ (catalog AF2997; R\&D Systems) antibodies. For Supplemental Figure 7 an antiHIF-1 $\alpha$ antibody from Novus Biologicals (catalog NB100-449) and a previously described anti-HIF- $2 \alpha$ antibody (43) were used.

ISH. Acta2, EGFP, Epo, Pdgfrb, Phd1, Phd3, and Vegf transcripts were detected on formalin-fixed, paraffin-embedded kidney tissue sections using the RNAscope Multiplex Fluorescent kit according to the manufacturer's instructions (Advanced Cell Diagnostics). Tissue slides were imaged on an Ariol SL-200 automated scanner (Leica Biosystems). Images were captured using the Leica Digital Image Hub and overlaid with a $100-\mu \mathrm{m}$ grid. Cells were counted in cortex and outer medulla. For each kidney section, cells were counted in 10 fields, each $0.16 \mathrm{~mm}^{2}$ in size. Cell counts were expressed as number of cells per square millimeter. For the analysis of human EPO and PDGFRB transcripts in kidneys, formalin-fixed, paraffin-embedded tissue sections were obtained from a total of 9 nephrectomy specimens (4 normal kidneys, 3 cases of hypertensive nephrosclerosis, 2 cases of nontumorous renal tissue from patients with renal cell cancer) and 5 human renal biopsy samples, and were processed as described above.

IHC. Formalin-fixed kidney sections were stained with polyclonal rabbit anti-HIF-1 $\alpha$ antiserum (catalog 10006421; Cayman Chemical Co.) at a dilution of 1:10,000, rabbit anti-HIF-2 $\alpha$ antiserum PM8, a gift from Peter Ratcliffe (University of Oxford, Oxford, UK) (54), at a dilution of 1:20,000, or rabbit anti-PDGFRB (catalog ab32570; Abcam) at a dilution of 1:500. HIF IHC was performed using CSA-II high signal amplification kit and Rabbit Link reagent (Dako) following the manufacturer's instructions. Slides were scanned and processed for imaging as described above.

Statistics. Data are reported as mean \pm SEM. Statistical analyses were performed with Prism 6 software (GraphPad Software Inc.) using Student's $t$ test and 1- or 2-way ANOVA with Tukey's post hoc analysis. $P$ values less than 0.05 were considered statistically significant.

Study approval. All studies involving human tissues were approved by the Institutional Review Boards of Vanderbilt University and Karolinska University Hospital. All procedures involving mice were performed in accordance with NIH guidelines for the use and care of live animals and were approved by the Vanderbilt University Institutional Animal Care and Use Committee.

\section{Author contributions}

HK and VHH conceived and designed the research studies, analyzed and interpreted data, wrote the manuscript, and made the figures. HK, QL, AAU, TCB, OD, PPK, and ASP performed experiments and acquired and analyzed data. HO, AW, ABF, GHF, and KWG contributed either mouse reagents or human tissues. KWG provided conceptual input. 


\section{Acknowledgments}

This work was supported by the Krick-Brooks chair in Nephrology (V.H. Haase), NIH grants R01-DK101791, R01-DK081646, and R01-DK080821, a Department of Veterans Affairs Merit Award (1I01BX002348) (all to V.H. Haase), and NIH grant R01-DK56942 (to A.B. Fogo). A. Wernerson is supported by the Regional Agreement on Medical Training and Clinical Research $(A L F)$ between Stockholm County Council and Karolinska Institutet. Additional support was provided by Vanderbilt's Diabetes Research and Training Center (P30-DK20593) and the Digestive Disease Research Center Cores (P30-DK058404). Whole slide imaging was performed in the Digital Histology Shared Resource at Vanderbilt University Medical Center (www. mc.vanderbilt.edu/dhsr). The authors wish to thank Peter Ratcliff and Tammie Bishop, University of Oxford, for generously providing the PM8 HIF- $2 \alpha$ antibody.

Address correspondence to: Volker H. Haase, Division of Nephrology and Hypertension, Vanderbilt University Medical Center, C-3119A MCN, 1161 21st Avenue, So., Nashville, Tennessee 37232-2372, USA. Phone: 615.343.7254; E-mail: volker.haase@ vanderbilt.edu.

Pinelopi P. Kapitsinou's present address is: Departments of Medicine and Anatomy and Cell Biology, and the Kidney Institute, University of Kansas Medical Center, Kansas City, Kansas, USA.
1. Koury MJ, Haase VH. Anaemia in kidney disease: harnessing hypoxia responses for therapy. Nat Rev Nephrol. 2015;11(7):394-410.

2. Scortegagna M, Morris MA, Oktay Y, Bennett M, Garcia JA. The HIF family member EPAS1/ HIF- $2 \alpha$ is required for normal hematopoiesis in mice. Blood. 2003;102(5):1634-1640.

3. Kapitsinou PP, et al. Hepatic HIF-2 regulates erythropoietic responses to hypoxia in renal anemia. Blood. 2010;116(16):3039-3048.

4. Semenza GL. Oxygen sensing, hypoxia-inducible factors, and disease pathophysiology. Annu Rev Pathol. 2014;9:47-71.

5. Kaelin WG Jr, Ratcliffe PJ. Oxygen sensing by metazoans: the central role of the HIF hydroxylase pathway. Mol Cell. 2008;30(4):393-402.

6. Koury ST, Bondurant MC, Koury MJ. Localization of erythropoietin synthesizing cells in murine kidneys by in situ hybridization. Blood. 1988;71(2):524-527.

7. Bachmann S, Le Hir M, Eckardt KU. Co-localization of erythropoietin mRNA and ecto-5'nucleotidase immunoreactivity in peritubular cells of rat renal cortex indicates that fibroblasts produce erythropoietin. J Histochem Cytochem. 1993;41(3):335-341.

8. Obara N, et al. Repression via the GATA box is essential for tissue-specific erythropoietin gene expression. Blood. 2008;111(10):5223-5232.

9. Paliege A, et al. Hypoxia-inducible factor- $2 \alpha-$ expressing interstitial fibroblasts are the only renal cells that express erythropoietin under hypoxia-inducible factor stabilization. Kidney Int 2010;77(4):312-318

10. Smith SW, Chand S, Savage CO. Biology of the renal pericyte. Nephrol Dial Transplant. 2012;27(6):2149-2155.

11. Gomez IG, Duffield JS. The FOXD1 lineage of kidney perivascular cells and myofibroblasts: functions and responses to injury. Kidney Int Suppl. 2014;4(1):26-33.

12. Koury ST, Koury MJ, Bondurant MC, Caro J, Graber SE. Quantitation of erythropoietin-producing cells in kidneys of mice by in situ hybridization: correlation with hematocrit, renal erythropoietin mRNA, and serum erythropoietin concentration. Blood.1989;74(2):645-651.

13. Kaissling B, Spiess S, Rinne B, Le Hir M. Effects of anemia on morphology of rat renal cortex. Am JPhysiol. 1993;264(4 pt 2):F608-F617.
14. Asada N, et al. Dysfunction of fibroblasts of extrarenal origin underlies renal fibrosis and renal anemia in mice. JClin Invest. 2011;121(10):3981-3990.

15. Humphreys BD, et al. Fate tracing reveals the pericyte and not epithelial origin of myofibroblasts in kidney fibrosis. Am J Pathol. 2010;176(1):85-97.

16. Kobayashi A, et al. Identification of a multipotent self-renewing stromal progenitor population during mammalian kidney organogenesis. Stem Cell Reports. 2014;3(4):650-662.

17. Muzumdar MD, Tasic B, Miyamichi K, Li L, Luo L. A global double-fluorescent Cre reporter mouse. Genesis. 2007;45(9):593-605.

18. Zeigler BM, Vajdos J, Qin W, Loverro L, Niss K. A mouse model for an erythropoietin-deficiency anemia. Dis Model Mech. 2011;3(11-12):763-772.

19. Takeda K, et al. Regulation of adult erythropoiesis by prolyl hydroxylase domain proteins. Blood. 2008;111(6):3229-3235.

20. Minamishima YA, et al. Somatic inactivation of the PHD2 prolyl hydroxylase causes polycythemia and congestive heart failure. Blood 2008;111(6):3236-3244.

21. Minamishima YA, Kaelin WG Jr. Reactivation of hepatic EPO synthesis in mice after PHD loss. Science. 2010;329(5990):407.

22. Querbes W, et al. Treatment of erythropoietin deficiency in mice with systemically administered siRNA. Blood. 2012;120(9):1916-1922.

23. Tojo Y, et al. Hypoxia signaling cascade for erythropoietin production in hepatocytes. Mol Cell Biol. 2015;35(15):2658-2672.

24. Liu J, et al. Cell-specific translational profiling in acute kidney injury. J Clin Invest. 2014;124(3):1242-1254.

25. Pan $X$, et al. Isolation and characterization of renal erythropoietin-producing cells from genetically produced anemia mice. PLoS One. 2011;6(10):e25839.

26. Armulik A, Genové G, Betsholtz C. Pericytes: developmental, physiological, and pathological perspectives, problems, and promises. Dev Cell. 2011;21(2):193-215

27. Lin SL, Kisseleva T, Brenner DA, Duffield JS. Pericytes and perivascular fibroblasts are the primary source of collagen-producing cells in obstructive fibrosis of the kidney. Am J Pathol. 2008;173(6):1617-1627.

28. Souma T, et al. Erythropoietin synthesis in renal myofibroblasts is restored by activation of hypoxia signaling. J Am Soc Nephrol. 2016;27(2):428-438.

29. Souma T, et al. Plasticity of renal erythropoietinproducing cells governs fibrosis. J Am Soc Nephrol. 2013;24(10):1599-1616.

30. Blumberg A, Keller H, Marti HR. Effect of altitude on erythropoiesis and oxygen affinity in anaemic patients on maintenance dialysis. Eur J Clin Invest. 1973;3(2):93-97.

31. Bosman DR, et al. Erythropoietin response to hypoxia in patients with diabetic autonomic neuropathy and non-diabetic chronic renal failure. Diabet Med. 2002;19(1):65-69.

32. Bernhardt WM, et al. Inhibition of prolyl hydroxylases increases erythropoietin production in ESRD. J Am Soc Nephrol. 2010;21(12):2151-2156.

33. Brookhart MA, et al. The effect of altitude on dosing and response to erythropoietin in ESRD. J Am Soc Nephrol. 2008;19(7):1389-1395.

34. Fishbane S, Nissenson AR. The new FDA label for erythropoietin treatment: how does it affect hemoglobin target? Kidney Int. 2007;72(7):806-813.

35. Unger EF, Thompson AM, Blank MJ, Temple R. Erythropoiesis-stimulating agents - time for a reevaluation. N Engl JMed. 2010;362(3):189-192.

36. Vaziri ND, Zhou XJ. Potential mechanisms of adverse outcomes in trials of anemia correction with erythropoietin in chronic kidney disease. Nephrol Dial Transplant. 2009;24(4):1082-1088.

37. Weidemann A, et al. The glial cell response is an essential component of hypoxiainduced erythropoiesis in mice. J Clin Invest. 2009;119(11):3373-3383.

38. Rankin EB, et al. The HIF signaling pathway in osteoblasts directly modulates erythropoiesis through the production of EPO. Cell. 2012;149(1):63-74.

39. Kurt B, et al. Deletion of von Hippel-Lindau protein converts renin-producing cells into erythropoietin-producing cells. J Am Soc Nephrol. 2013;24(3):433-444.

40. Kurt B, Gerl K, Karger C, Schwarzensteiner I, Kurtz A. Chronic hypoxia-inducible transcription factor-2 activation stably transforms juxtaglomerular renin cells into fibroblast-like cells in vivo. J Am Soc Nephrol. 2015;26(3):587-596.

41. Kishore BK, Isaac J, Westenfelder C. Administration of poly-D-glutamic acid induces proliferation of erythropoietin-producing peritubular cells in rat kidney. Am J Physiol Renal Physiol. 
2007;292(2):F749-F761.

42. Yamagishi S, et al. Vascular endothelial growth factor acts as a pericyte mitogen under hypoxic conditions. Lab Invest. 1999;79(4):501-509.

43. Rankin EB, et al. Hypoxia-inducible factor-2 (HIF-2) regulates hepatic erythropoietin in vivo. JClin Invest. 2007;117(4):1068-1077.

44. Kunze R, et al. Neuron-specific prolyl-4hydroxylase domain 2 knockout reduces brain injury after transient cerebral ischemia. Stroke. 2012;43(10):2748-2756.

45. Appelhoff RJ, et al. Differential function of the prolyl hydroxylases PHD1, PHD2, and PHD3 in the regulation of hypoxia-inducible factor. J Biol Chem. 2004;279(37):38458-38465.

46. Minamishima YA, et al. A feedback loop involv- ing the Phd3 prolyl hydroxylase tunes the mammalian hypoxic response in vivo. Mol Cell Biol. 2009;29(21):5729-5741.

47. Lubbers DW, Baumgartl H. Heterogeneities and profiles of oxygen pressure in brain and kidney as examples of the $\mathrm{pO} 2$ distribution in the living tissue. Kidney Int. 1997;51(2):372-380.

48. Takeda K, et al. Placental but not heart defects are associated with elevated hypoxia-inducible factor $\alpha$ levels in mice lacking prolyl hydroxylase domain protein 2. Mol Cell Biol. 2006;26(22):8336-8346.

49. Gruber M, et al. Acute postnatal ablation of Hif-2 $\alpha$ results in anemia. Proc Natl Acad Sci US A. 2007;104(7):2301-2306.

50. Liu Q, Davidoff O, Niss K, Haase VH. Hypoxiainducible factor regulates hepcidin via eryth- ropoietin-induced erythropoiesis. J Clin Invest. 2012;122(12):4635-4644.

51. Zhu X, Bergles DE, Nishiyama A. NG2 cells generate both oligodendrocytes and gray matter astrocytes. Development. 2008;135(1):145-157.

52. Foo SS, et al. Ephrin-B2 controls cell motility and adhesion during blood-vessel-wall assembly. Cell. 2006;124(1):161-173.

53. Kobayashi H, et al. Myeloid cell-derived hypoxiainducible factor attenuates inflammation in unilateral ureteral obstruction-induced kidney injury. J Immunol. 2012;188(10):5106-5115.

54 . Wiesener MS, et al. Widespread hypoxiainducible expression of HIF-2alpha in distinct cell populations of different organs. FASEB J. 2003;17(2):271-273. 